\title{
Invariant relations and Aschbacher classes of finite linear groups ${ }^{* \dagger}$
}

\author{
Jing $\mathrm{Xu}^{\ddagger}$ Michael Giudici Cai Heng Li Cheryl E. Praeger \\ School of Mathematics and Statistics \\ The University of Western Australia \\ Crawley, WA 6009, Australia
}

Submitted: June 9, 2011; Accepted: Nov 11, 2011; Published: Nov 21, 2011

Mathematics Subject Classification: 20B05, 20B15, 20G40

\begin{abstract}
For a positive integer $k$, a $k$-relation on a set $\Omega$ is a non-empty subset $\Delta$ of the $k$-fold Cartesian product $\Omega^{k} ; \Delta$ is called a $k$-relation for a permutation group $H$ on $\Omega$ if $H$ leaves $\Delta$ invariant setwise. The $k$-closure $H^{(k)}$ of $H$, in the sense of Wielandt, is the largest permutation group $K$ on $\Omega$ such that the set of $k$-relations for $K$ is equal to the set of $k$-relations for $H$. We study $k$-relations for finite semi-linear groups $H \leq \Gamma \mathrm{L}(d, q)$ in their natural action on the set $\Omega$ of non-zero vectors of the underlying vector space. In particular, for each Aschbacher class $\mathcal{C}$ of geometric subgroups of $\Gamma \mathrm{L}(d, q)$, we define a subset $\operatorname{Rel}(\mathcal{C})$ of $k$-relations (with $k=1$ or $k=2$ ) and prove (i) that $H$ lies in $\mathcal{C}$ if and only if $H$ leaves invariant at least one relation in $\operatorname{Rel}(\mathcal{C})$, and (ii) that, if $H$ is maximal among subgroups in $\mathcal{C}$, then an element $g \in \Gamma \mathrm{L}(d, q)$ lies in the $k$-closure of $H$ if and only if $g$ leaves invariant a single $H$-invariant $k$-relation in $\operatorname{Rel}(\mathcal{C})$ (rather than checking that $g$ leaves invariant all $H$-invariant $k$-relations). Consequently both, or neither, of $H$ and $H^{(k)} \cap \Gamma \mathrm{L}(d, q)$ lie in $\mathcal{C}$. As an application, we improve a 1992 result of Saxl and the fourth author concerning closures of affine primitive permutation groups.
\end{abstract}

Keywords: closures of permutation groups, Aschbacher classes of linear groups, primitive permutation group

${ }^{*}$ This work forms part of the PhD project of the first author, supported by an IPRS scholarship of Australia. This project forms part of an ARC Discovery Project. The second, third and fourth authors are supported by an Australian Research Fellowship, QEII Fellowship, and Federation Fellowship, respectively.

$\dagger$ Emails: xujing@mail.cnu.edu.cn, michael.giudici@uwa.edu.au, cai.heng.li@uwa.edu.au, cheryl.praeger@uwa.edu.au.

${ }^{\ddagger}$ Jing Xu’s current address: Department of Mathematics, Capital Normal University, Beijing 100048, China.

THE ELECTRONIC JOURNAL OF COMBINATORICS 18 (2011), \#P225 


\section{Introduction}

Let $H$ be a group of semi-linear transformations of a finite vector space $V$. If $H$ is reducible, then it preserves a nonzero proper subspace of $V$; we can regard this as a unary relation preserved by $H$. Similarly, if $H$ preserves a symplectic form, up to scalars and field automorphisms, then $H$ preserves the binary relation of orthogonality on $V$ with respect to this form. The aim of this paper is to determine similar unary or binary invariant relations that characterise each of the Aschbacher classes $\mathcal{C}_{1}, \ldots, \mathcal{C}_{8}$ of semi-linear groups. We do this in terms of natural geometric invariants. The Aschbacher classes are defined in Section 2.2 and the corresponding relations are given in Section 4, following a discussion of special cases in Section 3. We then apply our results to $k$-closures (in the sense of Wielandt [19]) of affine permutation groups, extending work of Jan Saxl and the fourth author [14].

More formally, for a positive integer $k$, a $k$-relation on a set $\Omega$ is a non-empty subset of $\Omega^{k}=\overbrace{\Omega \times \cdots \times \Omega}^{k}$, and for $H \leq \operatorname{Sym}(\Omega)$, the set of $H$-invariant $k$-relations is denoted $\operatorname{Rel}(H, k)$. The $k$-closure $H^{(k)}$ of a permutation group $H \leq \operatorname{Sym}(\Omega)$ is the largest subgroup of $\operatorname{Sym}(\Omega)$ with the same set of invariant $k$-relations as $H$, and Wielandt [19] noted that if $k>k^{\prime}$ then $H \leq H^{(k)} \leq H^{\left(k^{\prime}\right)}$.

In this paper we consider subgroups of $\Gamma \mathrm{L}(d, q)$ lying in certain classes $\mathcal{C}_{i}$, for $i \in$ $\{1, \ldots, 7, \mathbf{S p}, \mathbf{U}, \mathbf{O}\}$, which are defined in Subsection 2.2 and are similar to the classes in Aschbacher's classification [1]. For each $i$, we define an integer $k_{i} \in\{1,2\}$ and a set $\operatorname{Rel}\left(i, k_{i}\right)$ of $k_{i}$-relations on $\Omega$. The definitions of the $k_{i}$ and references to the definitions of $\operatorname{Rel}\left(i, k_{i}\right)$, given in Section 4, are summarised in Table 1. We prove that membership of a subgroup in the class $\mathcal{C}_{i}$ is equivalent to invariance of some relation in the relation set $\operatorname{Rel}\left(i, k_{i}\right)$.

\begin{tabular}{l|ccccccc}
\hline$i$ & 1 & 2 & 3 & 4 & 5 & 6 & 7 \\
$k_{i}$ & 1 & 1 & 2 & 1 & 1 & 2 & 1 \\
Definitions & $(4.1 .1)$ & $(4.2 .1)$ & $(4.3 .1)$ & $(4.4 .2)$ & $(4.5 .1)$ & $(4.6 .2)$ & $(4.4 .4)$ \\
\hline$i$ & $\mathbf{S p}$ & $\mathbf{U}$ & $\mathbf{O}$ & & & & \\
$k_{i}$ & 2 & 1 & 1 & & & & \\
Definitions & $(4.7 .1)$ & $(4.7 .4)$ & $(4.7 .3)$ & & & & \\
\hline
\end{tabular}

Table 1: References for definitions of the relation sets $\operatorname{Rel}\left(i, k_{i}\right)$.

Theorem 1.1. Let $d \geq 2, H \leq \Gamma \mathrm{L}(d, q), i \in\{1, \ldots, 7, \mathbf{S p}, \mathbf{U}, \mathbf{O}\}$, and $k_{i}$ be as in Table 1 . Then $H \in \mathcal{C}_{i}$ if and only if $\operatorname{Rel}\left(H, k_{i}\right) \cap \operatorname{Rel}\left(i, k_{i}\right) \neq \emptyset$.

This result has a number of important consequences, including a broad-brush result for linear groups, concerning their 'Aschbacher types' and the types of their $k_{i}$-closures.

Corollary 1.2. Let $H, i$ and $k_{i}$ be as in Theorem 1.1 and let $g \in \Gamma \mathrm{L}(d, q)$. Then the following all hold. 
(a) If $H \in \mathcal{C}_{i}$ and $g$ leaves invariant some relation in $\operatorname{Rel}\left(H, k_{i}\right) \cap \operatorname{Rel}\left(i, k_{i}\right)$, then also $\langle H, g\rangle \in \mathcal{C}_{i}$.

(b) $H \in \mathcal{C}_{i}$ if and only if $H^{\left(k_{i}\right)} \cap \Gamma \mathrm{L}(d, q) \in \mathcal{C}_{i}$.

(c) If $H$ is a maximal $\mathcal{C}_{i}$-subgroup then $H^{\left(k_{i}\right)} \cap \Gamma \mathrm{L}(d, q)=H$.

Thus, for a maximal $\mathcal{C}_{i}$-subgroup $H$, membership of $g \in \Gamma \mathrm{L}(d, q)$ in $H^{\left(k_{i}\right)}$ can be guaranteed if $g$ preserves a single relation in $\operatorname{Rel}\left(i, k_{i}\right) \cap \operatorname{Rel}(H, i)$, (rather than needing to check that $g$ preserves every $k_{i}$-relation in $\operatorname{Rel}\left(H, k_{i}\right)$ ).

Remark 1.3. For completeness we give information, in Section 3, about Wielandt closures in the cases not covered by Corollary 1.2. In terms of the notation for the Frobenius automorphism introduced in Subsection 2.1, we prove in Proposition 3.1.1 that, if $d=1$ then $H^{(2)}=H$; and in Proposition 3.2.1 that, if $H$ contains $\operatorname{SL}(d, q)$, then $H^{(2)} \cap \Gamma \mathrm{L}(d, q)$ is $\operatorname{GL}(d, q) \rtimes\langle\tau\rangle$ if $d \geq 3$, or is contained in $H\left\langle\tau^{j}\right\rangle$ if $d=2$, where $\left\langle\tau^{j}\right\rangle=\{\tau(h) \mid h \in H\}$. Finally we prove in Proposition 3.3.1 that if $H \in \mathcal{C}_{9}$ (defined in Subsection 2.2), then either $H^{(2)} \cap \Gamma \mathrm{L}(d, q) \in \mathcal{C}_{9}$ also, or $H=A_{7}<\mathrm{GL}(4,2)<H^{(2)}=A_{15}$.

This investigation was inspired by the 1992 paper [14] of Jan Saxl and the fourth author studying the $k$-closures of primitive permutation groups $G$ on a finite set $\Omega$. It was shown in [14] that, for $k \geq 2$, either $G$ and $G^{(k)}$ have the same socle, or their socles are known explicitly. (The socle of a group is the product of its minimal normal subgroups.) In the case of an affine primitive group $G$ the socle is an elementary abelian $p$-group, say $N=Z_{p}^{d}$, and $G=N H$ with $H$ an irreducible subgroup of $\operatorname{GL}(d, p)$, for some prime $p$ and $d \geq 1$. Thus, knowing that $G^{(k)}$ has socle $N$ in this case is a rather weak conclusion. The authors of [14] asked whether more information could be given about closures of finite affine primitive groups. An application of our main Theorem 1.1 provides such additional information for the 3-closures. All the proofs up to this point use elementary group theoretic and geometric methods. However, in making this application we use the finite simple group classification to determine (more precisely than in [14]) all the affine primitive groups $G$ for which $G^{(3)}$ is not affine.

Theorem 1.4. Suppose that $G$ is an affine primitive permutation group such that $G=$ $N H$ with $N=Z_{p}^{d}$ and $H \leq \operatorname{GL}(d, p)$, where $d \geq 1$ and $p$ is a prime. Then either

(a) [non-affine] $G^{(3)}$ is not an affine group, $p=2$, and if $G<L \leq G^{(3)}$ and $L$ is not an affine group, then $H, L$ are as in one of the lines of Table 2 , or

(b) [affine] $G^{(3)}=N K$ with $K \leq \mathrm{GL}(d, p)$ and one of the following holds.

(i) $d=1$ or 2 and $G^{(3)}=G$,

(ii) $d \geq 3, p$ is odd, and $\operatorname{SL}(d, p) \leq H \leq K \leq \mathrm{GL}(d, p)$,

(iii) $d \geq 3$ and, for some $i \in\{1, \ldots, 7, \mathbf{S p}, \mathbf{U}, \mathbf{O}\}$, both $H, K \in \mathcal{C}_{i}$, and $\operatorname{Rel}\left(K, k_{i}\right) \cap$ $\operatorname{Rel}\left(i, k_{i}\right) \neq \emptyset$, with $\operatorname{Rel}\left(i, k_{i}\right)$ as in Table 1 , 


\begin{tabular}{c|c|c}
\hline$d$ & $H$ & $L$ \\
\hline$\geq 3$ & $\mathrm{GL}(d, 2)$ & $A_{2^{d}}$ or $S_{2^{d}}$ \\
4 & $A_{7}$ & $A_{16}$ or $S_{16}$ \\
$n m$ & $\mathrm{GL}(n, 2) \imath Y$ & $A_{2^{n}} \imath Y \leq L \leq G^{(3)} \leq S_{2^{n}} \imath S_{m}$ \\
$4 m$ & $A_{7} 2 Y$ & $A_{16} \imath Y \leq L \leq G^{(3)} \leq S_{16} 2 S_{m}$ \\
\hline
\end{tabular}

Table 2: Result table for Theorem 1.4(a). In Lines 3 and $4, m \geq 2, n \geq 3$ and $Y \leq S_{m}$ is transitive.

(iv) $d \geq 3$, both $H, K \in \mathcal{C}_{9}$, but $(d, p, H) \neq\left(4,2, A_{7}\right)$.

Acknowledgements We thank an anonymous referee for helpful comments which improved the exposition of the paper.

\section{Preliminaries}

\section{$2.1 \quad$ Semi-linear transformations}

Throughout the rest of the paper, let $V=V(d, q)$ be a vector space of dimension $d \geq 1$ over a finite field $F_{q}$ of order $q$, where $q=p^{f}$ with $p$ a prime and $f \geq 1$. Also let $\Omega=V \backslash\{0\}$, and let $Z$ denote the subgroup of non-zero scalar transformations of $V$, so $Z \cong F_{q}^{*}$. Suppose that $H \leq \Gamma \mathrm{L}(d, q)$, so that $H$ acts on $\Omega$ faithfully.

Pick a basis $\left\{v_{1}, \ldots, v_{d}\right\}$ of $V$ and use it to identify $V$ with $F_{q}^{d}$. Let $\tau$ denote the Frobenius automorphism of $F_{q}$, that is, $\tau: \lambda \rightarrow \lambda^{p}$ for each $\lambda \in F_{q}$. We define an action of $\tau$ on $\Omega$ as follows: $\left(\lambda_{1} v_{1}+\ldots+\lambda_{d} v_{d}\right)^{\tau}=\lambda_{1}^{\tau} v_{1}+\ldots+\lambda_{d}^{\tau} v_{d}=\lambda_{1}^{p} v_{1}+\ldots+\lambda_{d}^{p} v_{d}$ for $\lambda_{i} \in F_{q}$. Then $\Gamma \mathrm{L}(d, q)=\mathrm{GL}(d, q) \rtimes\langle\tau\rangle$, the group of semi-linear transformations of $V$. In the following discussion, when we say 'the Frobenius automorphism $\tau \in \Gamma \mathrm{L}(d, q)$ ', $\tau$ will always be defined as above with respect to a specified basis.

For any $h \in \Gamma \mathrm{L}(d, q)=\mathrm{GL}(d, q) \rtimes\langle\tau\rangle$, let $\tau(h)$ be the associated field automorphism, that is, $\tau(h) \in\langle\tau\rangle$ and

$$
(\lambda v)^{h}=\lambda^{\tau(h)} v^{h} \quad \text { for any } v \in V \text { and } \lambda \in F_{q} .
$$

Then $\tau(h)$ is well defined (independently of the basis $\left\{v_{1}, \ldots, v_{d}\right\}$ ). Moreover, $\tau(h)=\tau^{j}$ for some integer $j$ satisfying $0 \leq j<f$, and $\tau\left(h_{1} h_{2}\right)=\tau\left(h_{1}\right) \tau\left(h_{2}\right)$.

\subsection{Aschbacher's classification}

As we indicated in Section 1, our proof of Theorem 1.1 is based on Aschbacher's description of subgroups of $\Gamma \mathrm{L}(d, q)$ not containing $\mathrm{SL}(d, q)$, (see [1] and [11]). Let $V, Z$ be as above. The families of subgroups $\mathcal{C}_{1}, \ldots, \mathcal{C}_{9}$ of $\Gamma \mathrm{L}(d, q)$ are described as follows. Because the groups behave differently in our investigations, we subdivide the class $\mathcal{C}_{8}$ as $\mathcal{C}_{8}=\mathcal{C}_{\mathbf{S p}} \cup$ $\mathcal{C}_{\mathbf{U}} \cup \mathcal{C}_{\mathbf{O}}$ 
$\mathcal{C}_{1}$ : These subgroups act reducibly on $V$, and maximal subgroups in this family are the stabilizers of proper non-trivial $F_{q}$-subspaces.

$\mathcal{C}_{2}$ : These subgroups act irreducibly but imprimitively on $V$, and maximal subgroups in this family are the stabilizers of direct sum decompositions $V=\oplus_{i=1}^{t} V_{i}$, where $t \geq 2$ and, for each $i, \operatorname{dim} V_{i}=d / t$.

$\mathcal{C}_{3}$ : These subgroups preserve on $V$ the structure of a vector space over an extension field $F_{q^{b}}$ of $F_{q}$, for some divisor $b$ of $d$ with $b>1$, and a maximal subgroup in this family, relative to a fixed value of $b$, is the stabilizer of a $d / b$-dimensional vector space structure on $V$ over the extension field $F_{q^{b}}$.

$\mathcal{C}_{4}$ : These subgroups preserve on $V$ the structure of a tensor product of subspaces, and maximal subgroups in this family are the stabilizers of tensor decompositions $V=V_{1} \otimes V_{2}$ such that $\operatorname{dim} V_{i} \geq 2$ for $i=1,2$ and $\operatorname{dim} V_{1} \neq \operatorname{dim} V_{2}$.

$\mathcal{C}_{5}$ : These subgroups preserve, modulo scalars, a structure on $V$ of a vector space over a proper subfield $F_{q_{0}}$ of $F_{q}$, where $q_{0}=p^{f / b}$ for some divisor $b>1$ of $f$. A maximal subgroup in this family, relative to a fixed value of $b$, is a central product of the scalar subgroup $Z$ and the stabilizer of a $d$-dimensional $F_{q_{0}}$-subspace of $V$.

$\mathcal{C}_{6}$ : These subgroups have as a normal subgroup an $r$-group $R$ of symplectic type (where $r$ is a prime, $r \neq p$, and $d$ is a power of $r$ ), $R$ acts absolutely irreducibly on $V$, and maximal subgroups in this family are the normalizers of these subgroups.

$\mathcal{C}_{7}$ : These subgroups preserve on $V$ a tensor decomposition $V=\otimes_{i=1}^{t} V_{i}$ with $t \geq 2$ and each $\operatorname{dim} V_{i}=c$ where $d=c^{t}$, and maximal subgroups in this family are the stabilizers of such decompositions.

$\mathcal{C}_{8}$ : Here $\mathcal{C}_{8}=\cup_{\mathbf{X} \in \mathcal{X}} \mathcal{C}_{\mathbf{X}}$, where $\mathcal{X}=\{\mathbf{S p}, \mathbf{U}$, or $\mathbf{O}\}$, and $\mathcal{C}_{\mathbf{X}}$ consists of all subgroups that preserve modulo scalars a non-degenerate $\mathbf{X}$-form on $V$, namely a non-degenerate alternating, hermitian, or quadratic form according as $\mathbf{X}=\mathbf{S p}, \mathbf{U}, \mathbf{O}$ respectively. Maximal subgroups in $\mathcal{C}_{\mathbf{X}}$ are normalizers of the corresponding classical groups that stabilize such X-forms.

$\mathcal{C}_{9}$ : These subgroups $H$ are not contained in $\mathcal{C}_{i}$ for any $i=1, \ldots, 8$. In particular the action of $H$ on $V$ is absolutely irreducible, primitive, not definable over any proper subfield of $F_{q}$, etc., and $H$ does not preserve modulo scalars any non-degenerate sesquilinear or quadratic form. In addition, $d \geq 2$ and there is a nonabelian simple group $T$ such that $T \leq H /(H \cap Z) \leq \operatorname{Aut} T$.

Remark 2.2.1. (a) We have defined the classes $\mathcal{C}_{i}(i=1, \ldots, 8)$ as subgroups possessing a particular property. As a consequence some subgroups may belong to more than one class. For example, we include the normalizers of $\mathrm{SO}\left(2 m+1,2^{f}\right)$ as maximal $\mathcal{C}_{\mathbf{O}^{-}}$-subgroups as they are classical groups. In addition, they are $\mathcal{C}_{1}$-subgroups as they preserve the 1dimensional radicals of the associated non-degenerate quadratic forms. We allow these overlaps in all cases except in the case $d=2$ where stabilisers of quadratic forms modulo scalars are $\mathcal{C}_{3}$-subgroups: we will not consider such groups as $\mathcal{C}_{8}$-groups. See also Section 4.7 .

(b) Aschbacher's Theorem [1] may be viewed as the assertion that, if $d \geq 2$, then every subgroup of $\Gamma \mathrm{L}(d, q)$ not containing $\operatorname{SL}(d, q)$ lies in at least one of the classes $\mathcal{C}_{1}, \ldots, \mathcal{C}_{9}$. 
Aschbacher's Theorem also applies to analogous classes of the finite classical groups, and we use the version for classical groups in the proof of Lemma 4.6.8.

(c) If $d=1$ the only non-empty Aschbacher classes are $\mathcal{C}_{5}$ (if $f>1$ ) and $\mathcal{C}_{\mathbf{O}}$ (if $q$ is odd), and even in these cases the maximal $\mathcal{C}_{i}$-subgroup is the whole group $\Gamma \mathrm{L}(1, q)$. The only assertions claimed in Section 1 for this case are those in Theorem 1.4 related to affine primitive groups. These assertions, and more, follow from Proposition 3.1 and an application of Lemma 2.3.1(4).

\subsection{General results about $k$-closures}

Let $G \leq \operatorname{Sym}(\Omega)$ be a permutation group on a set $\Omega$ of $n$ points, and let $k$ be a positive integer. Then $G$ has a natural action on $\Omega^{k}=\Omega \times \cdots \times \Omega$ (k copies). From the definition of the $k$-closure $G^{(k)}$ in Section 1 we see that

$$
G^{(k)}:=\left\{g \in \operatorname{Sym}(\Omega) \mid \Delta^{g}=\Delta \text { for each orbit } \Delta \text { of } G \text { on } \Omega^{k}\right\} .
$$

This implies that, for $k \geq 2, G \leq \ldots \leq G^{(k+1)} \leq G^{(k)} \leq \ldots \leq G^{(2)}$. We say that $G$ is $k$-closed if $G=G^{(k)}$. Recall that $\operatorname{Rel}(G, k)$ is the set of all $G$-invariant $k$-relations on $\Omega$. For $L \leq \operatorname{Sym}(\Omega)$, we say that $G$ is $k$-equivalent to $L$ if $\operatorname{Rel}(G, k)=\operatorname{Rel}(L, k)$. This condition is equivalent to the condition that $G$ and $L$ have the same orbit set on $\Omega^{k}$. In particular, $G$ is $k$-equivalent to $G^{(k)}$.

We collect some useful fundamental results here. Proofs may be found in the Lecture Notes of Wielandt [19]. The proof of Lemma 2.3.1 (1), (2), (3) and (4) can be found in Theorems 5.8, 5.7, 5.12, 4.3 and Lemma 4.12 of [19] respectively.

Lemma 2.3.1. [19, Wielandt] Let $k \geq 1$ and let $G$ and $L$ be permutation groups on a set $\Omega$. Then

(1) $G \leq G^{(k+1)} \leq G^{(k)}$.

(2) If $G \leq L$, then $G^{(k)} \leq L^{(k)}$.

(3) If there exist $\alpha_{1}, \ldots, \alpha_{k} \in \Omega$ such that $G_{\alpha_{1}, \ldots, \alpha_{k}}=1$, then $G^{(k+1)}=G$.

(4) If $G$ is $(k+1)$-equivalent to $L$, then $G$ is $k$-equivalent to $L$ and for any $\alpha \in \Omega, G_{\alpha}$ is $k$-equivalent to $L_{\alpha}$.

The following lemma is an easy result about the $k$-closure of an induced quotient action.

Lemma 2.3.2. Suppose $k \geq 1$ and $G, L \leq \operatorname{Sym}(\Omega)$. Suppose further that $G$ is $k$-equivalent to $L$ on $\Omega$. Let $N$ be an intransitive normal subgroup of both $G$ and $L$. Let $\bar{\Omega}$ be the set of $N$-orbits. Then $\bar{G}=G / N$ is k-equivalent to $\bar{L}=L / N$ on $\bar{\Omega}$.

Proof. For $\alpha \in \Omega$, let $[\alpha]$ denote the $N$-orbit containing $\alpha$. Suppose $\left(\left[\alpha_{1}\right], \ldots,\left[\alpha_{k}\right]\right) \in \bar{\Omega}^{k}$. For any $\bar{x}=x N \in \bar{L}$ where $x \in L$, the normality of $N$ implies that $\left(\left[\alpha_{1}\right], \ldots,\left[\alpha_{k}\right]\right)^{\bar{x}}=$ $\left(\left[\alpha_{1}^{x}\right], \ldots,\left[\alpha_{k}^{x}\right]\right)$. Since $G$ is $k$-equivalent to $L$ on $\Omega$, there exists $g \in G$ such that $\left(\alpha_{1}^{x}, \ldots, \alpha_{k}^{x}\right)=$ $\left(\alpha_{1}^{g}, \ldots, \alpha_{k}^{g}\right)$. Hence $\left(\left[\alpha_{1}\right], \ldots,\left[\alpha_{k}\right]\right)^{\bar{x}}=\left(\left[\alpha_{1}\right], \ldots,\left[\alpha_{k}\right]\right)^{\bar{g}}$ where $\bar{g}=g N \in \bar{G}$. Therefore $\bar{G}$ is $k$ equivalent to $\bar{L}$ on $\bar{\Omega}$. 


\subsection{Dickson's Theorem}

When we handle the subgroups of $\operatorname{GL}(2, q)$, the 1901 classification by L. E. Dickson [4] of the subgroups of $\operatorname{PSL}(2, q)$ is one of our main tools (see [17, Chapter 3, §6] or [7, Chaper $2, \S 8]$ for a proof).

Theorem 2.4.1. [Dickson] Let $q=p^{f}$, where $p$ is a prime and $f \geq 1$, and let $s=$ $\operatorname{gcd}(2, q-1)$. Also let $z$ be an integer dividing $\frac{q+1}{s}$ or $\frac{q-1}{s}$. Then a subgroup of $\operatorname{PSL}(2, q)$ is isomorphic to one of the following groups:

(a) an elementary abelian p-group $Z_{p}^{m}$, where $1 \leq m \leq f$;

(b) a cyclic group of order $z$;

(c) a dihedral group of order $2 z$;

(d) $A_{4}$ if $p$ is odd;

(e) $S_{4}$ if $p^{2 f}-1 \equiv 0(\bmod 16)$;

(f) $A_{5}$ if $p^{2 f}-1 \equiv 0(\bmod 10)$;

(g) $Z_{p}^{m} \rtimes Z_{t}$ where $m \leq f, t \mid \frac{p^{m}-1}{s}$ and $t \mid\left(p^{f}-1\right)$;

(h) $\operatorname{PSL}\left(2, p^{m}\right)$ if $m \mid f$, or $\operatorname{PGL}\left(2, p^{m}\right)$ if $2 m \mid f$.

\subsection{Primitive permutation groups preserving a product decom- position}

A permutation group $G$ on $\Omega$ is said to preserve a product decomposition $\Gamma^{m}$ of $\Omega$, where $m \geq 2$, if $\Omega$ can be identified with the Cartesian product $\Gamma^{m}=\Gamma_{1} \times \ldots \times \Gamma_{m}\left(\right.$ with $\Gamma_{i}=\Gamma$ for $1 \leq i \leq m$ ) in such a way that $G$ is a subgroup of the wreath product

$$
W=\operatorname{Sym}(\Gamma) \imath S_{m}=\operatorname{Sym}(\Gamma)^{m} \rtimes S_{m}
$$

in product action. This means that, for $g=\left(g_{1}, \ldots, g_{m}\right)$ in the 'base group' $\operatorname{Sym}(\Gamma)^{m}$,

$$
\left(\gamma_{1}, \ldots, \gamma_{m}\right)^{g}=\left(\gamma_{1}^{g_{1}}, \ldots, \gamma_{m}^{g_{m}}\right)
$$

and for $t$ in the 'top group' $S_{m}$,

$$
\left(\gamma_{1}, \ldots, \gamma_{m}\right)^{t^{-1}}=\left(\gamma_{1^{t}}, \ldots, \gamma_{m^{t}}\right)
$$

where $\left(\gamma_{1}, \ldots, \gamma_{m}\right) \in \Omega=\Gamma^{m}$. Thus if $\alpha=(\delta, \ldots, \delta) \in \Omega$, then $W_{\alpha}=(\operatorname{Sym}(\Gamma))_{\delta} \imath S_{m}$.

The projection of $W=\operatorname{Sym}(\Gamma)^{m} \rtimes S_{m}$ onto $S_{m}$, which we denote by $\pi$, may be considered as a permutation representation of $W$ on $\{1, \ldots, n\}$. Then, for $1 \leq i \leq m$, the subgroup

$$
W_{i}=\operatorname{Sym}\left(\Gamma_{i}\right) \times\left(\operatorname{Sym}(\Gamma) \imath S_{m-1}\right)
$$

is the full preimage under $\pi$ of the stabilizer of $i$. Let $\pi_{i}$ denote the projection $W_{i} \rightarrow$ $\operatorname{Sym}\left(\Gamma_{i}\right)$ of $W_{i}$ onto the first factor of this direct product.

Now suppose that $G \leq W$ and $G$ is primitive on $\Omega=\Gamma^{m}$. The primitivity of $G$ implies that $Y:=\pi(G) \leq S_{m}$ is transitive. The subgroup $G \cap W_{i}$ consists of all the elements of $G$ which fix $i$, and the restriction of $\pi_{i}$ to $G \cap W_{i}$ is a homomorphism from $G \cap W_{i}$ onto 
a subgroup of $\operatorname{Sym}\left(\Gamma_{i}\right)$. Set $G_{0}:=\pi_{1}\left(G \cap W_{1}\right)$ and $\Gamma=\Gamma_{1}$ so that $G_{0} \leq \operatorname{Sym}(\Gamma)$. By a result of Kovacs $[12,2.2]$, replacing $G$ by a conjugate of $G$ under an element of $W$, if necessary, we may assume that

$$
G \leq G_{0} 2 S_{m}
$$

Moreover, see $[12,2.3], G_{0}$ is primitive on $\Gamma$ and not of prime order.

In summary, when dealing with primitive groups $G$ on $\Omega$ that preserve a product decomposition $\Omega=\Gamma^{m}$, we may assume that $G \leq G_{0}<Y$, where $Y=\pi(G) \leq S_{m}$ is transitive, and $G_{0}=\pi_{1}\left(G \cap W_{1}\right) \leq \operatorname{Sym}(\Gamma)$ is primitive and not of prime order. The group $G_{0}$ is called the group induced by $G$ on $\Gamma$.

\section{Proofs for special cases}

\subsection{1-dimensional semi-linear groups}

Let $q=p^{f}$ and $\Omega=V \backslash\{0\}$ as in Subsection 2.1 with $d=1$. As mentioned in Remark 2.2.1, when $d=1$ the only non-empty Aschbacher classes are $\mathcal{C}_{5}$ (if $f>1$ ) and $\mathcal{C}_{\mathbf{O}}$ (if $q$ is odd), and in these cases the unique maximal $\mathcal{C}_{i}$-subgroup is $\Gamma \mathrm{L}(1, q)$. As promised in Remark 1.3, we prove here that each subgroup $H$ of $\Gamma \mathrm{L}(1, q)$ is 2-closed. If $H=\Gamma \mathrm{L}(1, q)$ this fact and more follows from [15, Corollary 4.1]. Define the 2-relation $\Delta$ on $\Omega$ by:

$$
\Delta:=\left\{\left(x, x \xi^{p^{i}}\right) \mid x \in \Omega, 0 \leq i<f\right\}, \quad \text { where } \xi \text { is a primitive element of } F_{q} .
$$

Proposition 3.1.1. Let $G=\Gamma \mathrm{L}(1, q), H \leq G \leq \operatorname{Sym}(\Omega)$, and $g \in \operatorname{Sym}(\Omega)$. Then

(a) $g \in G^{(2)}$ if and only if $g$ leaves $\Delta$ invariant; and

(b) $H=H^{(2)}$.

Proof. Part (a) follows from [15, Corollary 4.1], and this implies in particular that $G=$ $G^{(2)}$. Then by Lemma 2.3.1(2), $H^{(2)} \leq G^{(2)}=G$. For a primitive element $\xi \in F_{q}$, the stabilizer in $G$ of the pair $(1, \xi)$ is trivial. By definition, $(1, \xi)^{H}=(1, \xi)^{H^{(2)}}$. Hence $|H|=\left|(1, \xi)^{H}\right|=\left|(1, \xi)^{H^{(2)}}\right|=\left|H^{(2)}\right|$, and so $H=H^{(2)}$.

Proposition 3.1.1 will also be used when considering groups $H$ of type $\mathcal{C}_{3}$ in Section 4 .

\subsection{The Case $\operatorname{SL}(d, q) \leq H \leq \Gamma L(d, q) \quad(d \geq 2)$}

Let $q=p^{f}, \Omega=V \backslash\{0\}, Z, \tau$ (defined relative to the basis $\left\{v_{1}, \ldots, v_{d}\right\}$ of $V$ ), as in Subsection 2.1. In this subsection we prove Proposition 3.2.1 and Proposition 3.2.2, as promised in Remark 1.3. Recall the definition of $\tau(h)$ for $h \in \mathrm{GL}(d, q)$ from (2.1.1).

Proposition 3.2.1. Suppose that $\mathrm{SL}(d, q) \leq H \leq \Gamma \mathrm{L}(d, q)$ with $d \geq 2$, and let $\left\langle\tau^{i}\right\rangle=$ $\{\tau(h) \mid h \in H\}$ and $K=H^{(2)} \cap \Gamma \mathrm{L}(d, q)$. Then either $d \geq 3$ and $K=\operatorname{GL}(d, q) \rtimes\left\langle\tau^{i}\right\rangle$, or $d=2$ and $H \leq K \leq H\left\langle\tau^{i}\right\rangle$. 
We see from Proposition 3.2.2 below that the case $d=2$ is really different from the general case of larger $d$. Proposition 3.2.2 both yields the second assertion of Proposition 3.2.1, and also shows, for example, that for $H=\operatorname{SL}(2, q)$ the subgroup $K=$ $H^{(2)} \cap \Gamma \mathrm{L}(2, q)$ is equal to $H$ (rather than $\left.\mathrm{GL}(2, q)\right)$. On the other hand we can sometimes have $K=\operatorname{GL}(d, q) \rtimes\left\langle\tau^{i}\right\rangle$ when $d=2$, see Example 3.2.3.

Proposition 3.2.2. Suppose that $H \leq \Gamma \mathrm{L}(2, q)$, and let $\left\langle\tau^{i}\right\rangle=\{\tau(h) \mid h \in H\}$ and $K=H^{(2)} \cap \Gamma \mathrm{L}(2, q)$. Then $K \leq H\left\langle\tau^{i}\right\rangle$, and in particular, if either $H \leq \mathrm{GL}(2, q)$ or $\tau^{i} \in H$, then $H=K$.

Proof. Let $\langle\xi\rangle=F_{q}^{*}$ and let $v \in \Omega=V \backslash\{0\}$. Since $(v, \xi v)^{H}=(v, \xi v)^{K}$, for any $g \in K$, there exists $h \in H$ such that $\left(v^{h},(\xi v)^{h}\right)=\left(v^{g},(\xi v)^{g}\right)$. Thus

$$
\xi^{\tau(h)} v^{h}=(\xi v)^{h}=(\xi v)^{g}=\xi^{\tau(g)} v^{g}=\xi^{\tau(g)} v^{h} .
$$

Therefore, $\tau(g)=\tau(h)$, and so $g h^{-1} \in K \cap \mathrm{GL}(2, q)$. Then $K=H(K \cap \operatorname{GL}(2, q))$.

Now for any $g \in \operatorname{GL}(2, q) \cap K, g$ is determined by the images of the basis vectors $v_{1}$ and $v_{2}$ under $g$. Since $\left(v_{1}, v_{2}\right)^{H}=\left(v_{1}, v_{2}\right)^{K}$, there exists $h \in H$ such that $\left(v_{1}^{g}, v_{2}^{g}\right)=\left(v_{1}^{h}, v_{2}^{h}\right)$. Thus $h=\tau(h) g$ and so $\tau(h)=h g^{-1} \in K$. It follows that $K \leq H\left\langle\tau^{i}\right\rangle$. Finally, if either $\tau^{i} \in H$ or $i=f$, then $K=H$.

Example 3.2.3. Let $F=F_{5^{2}}$ and $\langle\xi\rangle=F^{*} \cong Z_{24}$. Let det $\mathrm{GL}(2,25) \rightarrow F^{*}$ denote the determinant map det $: g \mapsto \operatorname{det}(g)$. Define

$$
H=\left\langle\mathrm{SL}(2,25), \tau g_{1}, g_{2}\right\rangle \quad \text { where } g_{1}=\left(\begin{array}{cc}
\xi^{3} & 0 \\
0 & 1
\end{array}\right) \text { and } g_{2}=\left(\begin{array}{cc}
\xi^{8} & 0 \\
0 & 1
\end{array}\right) \text {. }
$$

Then $\mathrm{SL}(2,25) \leq H \leq \Gamma \mathrm{L}(2,25)$ and $\langle\tau\rangle=\{\tau(h) \mid h \in H\}$. We claim that $H \neq \Gamma \mathrm{L}(2,25)$ and that $K=H^{(2)} \cap \Gamma \mathrm{L}(2,25)$ is equal to $\mathrm{GL}(2,25) \rtimes\langle\tau\rangle=\Gamma \mathrm{L}(2,25)$. (See Lemma 3.2.4.)

Lemma 3.2.4. The claims made in Example 3.2 .3 are true.

Proof. Now $\operatorname{det}\left(\left\langle g_{1}, g_{2}\right\rangle\right)=F^{*}$ and $\operatorname{det}\left(\left\langle g_{1}^{2}, g_{2}\right\rangle\right) \cong Z_{12}$, and in particular $\Gamma \mathrm{L}(2,25)=$ $\left\langle\mathrm{SL}(2,25), g_{1}, g_{2}, \tau\right\rangle$. Also, $\tau g_{1} \tau g_{1}=g_{1}^{\tau} g_{1}=\left(\begin{array}{cc}\xi^{18} & 0 \\ 0 & 1\end{array}\right)=g_{1}^{6}$, so that $\left\langle\left(\tau g_{1}\right)^{2}\right\rangle=\left\langle g_{1}^{6}\right\rangle=$ $\left\langle g_{1}^{2}\right\rangle$ and $H \cap \mathrm{GL}(2,25)=\left\langle\mathrm{SL}(2,25), g_{1}^{2}, g_{2}\right\rangle$. Thus $|H|=2|H \cap \mathrm{GL}(2,25)|=2(|\mathrm{SL}(2,25)|$. $12)=|\Gamma \mathrm{L}(2,25)| / 2$.

Let $L=\Gamma L(2,25)$ and consider $\Delta=\left(v_{1}, v_{2}\right)^{L}$. Then the stabilizer $L_{\left(v_{1}, v_{2}\right)}=\langle\tau\rangle$, and $\Delta=\left\{\left(w_{1}, w_{2}\right) \mid w_{1}, w_{2} \in \Omega\right.$ and $\left.w_{1} \notin\left\langle w_{2}\right\rangle\right\}$. Observe that $|\Delta|=|\operatorname{GL}(2,25)|=|H|$. Now since $\tau \notin H, H_{\left(v_{1}, v_{2}\right)}=1$ and so $\left|\left(v_{1}, v_{2}\right)^{H}\right|=|H|=|\Delta|$. Hence $\Delta$ is also an orbit of $H$. Also if $\Delta_{\lambda}=\{(v, \lambda v) \mid v \in \Omega\}$ where $\lambda \in F^{*}$, then $\Delta_{\lambda}^{H}=\Delta_{\lambda}^{L}=\Delta_{\lambda} \cup \Delta_{\lambda^{5}}$. Thus $L$ and $H$ have the same orbits in $\Omega \times \Omega$. Hence $L$ is 2-equivalent to $H$ on $\Omega$, so $L \leq H^{(2)}$.

Finally we prove Proposition 3.2.1.

Proof of Proposition 3.2.1. If $d=2$ the assertions have been proved already in Proposition 3.2.2, so suppose that $d \geq 3$. Then $\operatorname{SL}(d, q)$ is 2-equivalent to $\operatorname{GL}(d, q)$ as these two groups have the same orbit sets on $\Omega \times \Omega$, namely,

$$
\Delta=\{(v, w) \mid v, w \in \Omega \text { and } v \notin\langle w\rangle\} \quad \text { and } \quad \Delta_{\lambda}=\{(v, \lambda v) \mid v \in \Omega\} \text { where } \lambda \in F_{q}^{*} .
$$


Since each $H$-orbit in $\Omega \times \Omega$ is a union of $\operatorname{SL}(d, q)$-orbits, $\operatorname{GL}(d, q) \leq H^{(2)}$. Thus $\operatorname{GL}(d, q) \leq$ $K \leq \Gamma \mathrm{L}(d, q)=\mathrm{GL}(d, q) \rtimes\langle\tau\rangle$, and so $K=\mathrm{GL}(d, q) \rtimes\left\langle\tau^{j}\right\rangle$ for some integer $j$ dividing $f$.

Recall that $\left\langle\tau^{i}\right\rangle=\{\tau(h) \mid h \in H\}$. Then

$$
\Delta^{H}=\Delta^{\left\langle\tau^{i}\right\rangle}=\Delta \quad \text { and } \quad\left(\Delta_{\lambda}\right)^{H}=\left(\Delta_{\lambda}\right)^{\left\langle\tau^{i}\right\rangle}=\cup_{\mu \in \lambda\left\langle\tau^{i}\right\rangle} \Delta_{\mu}
$$

But if $\left\langle\tau^{i}\right\rangle \neq\left\langle\tau^{j}\right\rangle$, then there exists $\lambda \in F_{q}^{*}$ such that $\lambda^{\left\langle\tau^{i}\right\rangle} \neq \lambda^{\left\langle\tau^{j}\right\rangle}$. This would imply that $H$ is not 2-equivalent to $K=\operatorname{GL}(d, q) \rtimes\left\langle\tau^{j}\right\rangle$, which would be a contradiction. Hence $\left\langle\tau^{i}\right\rangle=\left\langle\tau^{j}\right\rangle$ and the result follows.

\subsection{The Case $H \in \mathcal{C}_{9}$}

Recall that $H \in \mathcal{C}_{9}$ if $H$ does not contain $\operatorname{SL}(d, q), d \geq 2$, and $H$ is not contained in any maximal $\mathcal{C}_{i}$-subgroup for $i=1,2, \ldots, 8$. In this subsection we identify the exceptional $\mathcal{C}_{9}$-group in Theorem 1.4(a), and prove some parts of Theorem 1.4 in Lemma 3.3.2.

Proposition 3.3.1. Suppose $H \in \mathcal{C}_{9}$ and let $K=H^{(2)} \cap \Gamma L(d, q)$. Then either $K \in \mathcal{C}_{9}$ or $(d, q, H)=\left(4,2, A_{7}\right)$.

Proof. By the definition of the class $\mathcal{C}_{9}$, and since $H \in \mathcal{C}_{9}$, it follows that either $K \in \mathcal{C}_{9}$ or $K \geq \operatorname{SL}(d, q)$. Assume the latter, and consider the natural action of $\operatorname{P\Gamma L}(d, q)$ on the set $\bar{\Omega}$ of 1-dimensional subspaces of $V$. By Lemma 2.3.2, $\bar{H}:=H Z / Z$ is 2-equivalent to $\bar{K}:=K Z / Z$ on $\bar{\Omega}$. By assumption $\bar{K} \geq \operatorname{PSL}(d, q)$, so $\bar{K}$ is 2 -transitive on $\bar{\Omega}$. Thus $\bar{H}$ is 2-transitive on $\bar{\Omega}$, and by the definition of the class $\mathcal{C}_{9}, \bar{H}$ does not contain $\operatorname{PSL}(d, q)$. If $d=2$ then by Theorem 2.4.1, $A_{5} \unlhd \bar{H} \leq S_{5}$ and $q^{2} \equiv 1(\bmod 10)$. In particular $q \geq 9$. However, since $\bar{H}$ is 2-transitive on $\bar{\Omega},(q+1) q$ must divide 120 , and this is impossible. Hence $d \geq 3$. By [2], $d=4, q=2$ and $H=A_{7}$, as in the statement.

Lemma 3.3.2. Suppose that $G=Z_{p}^{d} \cdot H$, with $H \leq \mathrm{GL}(d, p)$, and $G$ acts primitively on $V=V(d, p)$. If one of $d \leq 2$, or $\mathrm{SL}(d, p) \leq H$, or $H \in \mathcal{C}_{9}$, then the assertions made about such groups in Theorem 1.4 all hold.

Proof. If $d=1$, then $V=F_{p}$ and the stabilizer $G_{0,1}=1$. Hence by Lemma 2.3.1(3), $G^{(3)}=G$, as in Theorem 1.4(b)(i). So suppose that $d \geq 2$. If $p=2$ and either $H=$ $\operatorname{GL}(d, 2)$, or $d=4$ and $H=A_{7}$, then $G$ is 3 -transitive and hence $G^{(3)}=S_{2^{d}}$. It follows from [14, Lemma 4.1] that in these cases Theorem 1.4 holds (part (b)(i) if $d=2$, or part (a), Line 1 or 2 of Table 2 , if $d \geq 3$ ). In all other cases we have to consider here, $G$ is not 3-transitive.

It follows from [14, Theorem 2] that, in each of these remaining cases, $G^{(3)} \leq \mathrm{AGL}(d, p)$ and hence $G^{(3)}=Z_{p}^{d} \cdot K$ where $H \leq K \leq \mathrm{GL}(d, p)$. By Lemma 2.3.1 (4), $H$ and $K$ are 2-equivalent and so $H \leq K \leq H^{(2)} \cap \mathrm{GL}(d, p)$. If $d=2$, then by Proposition 3.2.2, $H^{(2)} \cap \mathrm{GL}(2, p)=H$ and hence $K=H$ and $G^{(3)}=G$, as in Theorem 1.4(b)(i) (and also in part (b)(iv) if $\left.H \in \mathcal{C}_{9}\right)$. We may assume now that $d \geq 3$ and $(d, p, H) \neq\left(4,2, A_{7}\right)$. If $H \in \mathcal{C}_{9}$ then, by Proposition 3.3.1, $H^{(2)} \cap \mathrm{GL}(d, p) \in \mathcal{C}_{9}$. In particular since $K \leq$ 
$H^{(2)} \cap \mathrm{GL}(d, p)$, it follows that $K$ does not contain $\mathrm{SL}(d, p)$. Since $H \leq K$, it follows from the definition of the class $\mathcal{C}_{9}$ that $K$ does not lie in $\mathcal{C}_{i}$ for any $i \leq 8$, and hence $K \in \mathcal{C}_{9}$, as in Theorem 1.4(b)(iv). Finally if $H \geq \mathrm{SL}(d, p)$ with $p$ odd and $d \geq 3$, then we have already proved that $G^{(3)} \leq \operatorname{AGL}(d, p)$ and $K \leq \operatorname{GL}(d, p)$, as in Theorem 1.4(b)(iii). We note in passing that a similar argument to that given in the proof of Proposition 3.2.1 would yield that $G^{(3)}=\operatorname{AGL}(d, p)$ in Theorem $1.4(\mathrm{~b})$ (iii) if $d \geq 4$. This however is not the case if, for example, $d=3$ and $H=\mathrm{SL}(3, p)$.

\section{Proof of Theorem 1.1}

Throughout Section 4, we use the notation of Subsection 2.1, and the definitions of the families $\mathcal{C}_{i}$ in Subsection 2.2, together with the following. Let $d \geq 2$, and $H \leq \Gamma \mathrm{L}(d, q)=$ $\mathrm{GL}(d, q) \rtimes\langle\tau\rangle$ such that $H \nsupseteq \mathrm{SL}(d, q)$. Let $i \in\{1,2, \ldots, 7, \mathbf{S p}, \mathbf{U}, \mathbf{O}\}$. We will define an integer $k_{i} \in\{1,2\}$, and a set $\operatorname{Rel}\left(i, k_{i}\right)$ of $k_{i}$-relations on $\Omega$, and prove that $H \in \mathcal{C}_{i}$ if and only if there exists an $H$-invariant relation in $\operatorname{Rel}\left(i, k_{i}\right)$. This will prove Theorem 1.1, and allow us to deduce Corollary 1.2 as follows.

Proof of Corollary 1.2.

(a) Suppose $H \in \mathcal{C}_{i}$ and $g \in \Gamma \mathrm{L}(d, q)$ leaves invariant some $\Delta \in \operatorname{Rel}\left(i, k_{i}\right) \cap \operatorname{Rel}\left(H, k_{i}\right)$. Then $\langle H, g\rangle$ leaves $\Delta$ invariant so $\Delta \in \operatorname{Rel}\left(i, k_{i}\right) \cap \operatorname{Rel}\left(\langle H, g\rangle, k_{i}\right)$. By Theorem 1.1, $\langle H, g\rangle \in \mathcal{C}_{i}$.

(b) By Theorem 1.1, $H \in \mathcal{C}_{i}$ if and only if $\operatorname{Rel}\left(i, k_{i}\right) \cap \operatorname{Rel}\left(H, k_{i}\right) \neq \emptyset$, and since by definition $\operatorname{Rel}\left(H, k_{i}\right)=\operatorname{Rel}\left(H^{\left(k_{i}\right)}, k_{i}\right)$, this holds if and only if $\operatorname{Rel}\left(i, k_{i}\right) \cap \operatorname{Rel}\left(H^{\left(k_{i}\right)} \cap\right.$ $\left.\Gamma \mathrm{L}(d, q), k_{i}\right) \neq \emptyset$. Finally, again by Theorem 1.1, this is true if and only if $H^{\left(k_{i}\right)} \cap \Gamma \mathrm{L}(d, q) \in$ $\mathcal{C}_{i}$

(c) Suppose that $H$ is a maximal $\mathcal{C}_{i}$-subgroup. By part (a), $H^{\left(k_{i}\right)} \cap \Gamma L(d, q) \in \mathcal{C}_{i}$ and contains $H$. By maximality, this subgroup is equal to $H$.

\subsection{The Case $H \in \mathcal{C}_{1}$}

Define

$$
k_{1}=1 \text { and } \operatorname{Rel}(1,1)=\{W \backslash\{0\} \mid W \text { is a non-zero proper subspace of } V\} .
$$

Since subgroups in $\mathcal{C}_{1}$ all leave invariant some non-zero proper subspace of $V$, Theorem 1.1 follows immediately for this case.

Proposition 4.1.1. $H \in \mathcal{C}_{1}$ if and only if $\operatorname{Rel}(H, 1) \cap \operatorname{Rel}(1,1) \neq \emptyset$.

\subsection{The Case $H \in \mathcal{C}_{2}$}

Define $k_{2}=1$ and

$$
\operatorname{Rel}(2,1)=\left\{\left(V_{1} \cup \ldots \cup V_{t}\right) \backslash\{0\} \mid V=V_{1} \oplus \cdots \oplus V_{t}, \quad d=a t, t>1, a=\operatorname{dim} V_{i}\right\} .
$$


Proposition 4.2.1. $H \in \mathcal{C}_{2}$ if and only if $\operatorname{Rel}(H, 1) \cap \operatorname{Rel}(2,1) \neq \emptyset$.

Proof. If $H$ is a $\mathcal{C}_{2}$-subgroup, then by definition there exists an $H$-invariant decomposition $V=V_{1} \oplus \cdots \oplus V_{t}$, where $d=a t, t>1$, and $a=\operatorname{dim} V_{i}$ for each $i$. The group $H$ leaves invariant the corresponding 1-relation in $\operatorname{Rel}(2,1)$.

Conversely, suppose $H$ leaves invariant the relation $\Delta=\left(V_{1} \cup \ldots \cup V_{t}\right) \backslash\{0\} \in \operatorname{Rel}(2,1)$. It is sufficient to prove that each $h \in H$ lies in the stabilizer $\operatorname{Stab}_{\Gamma L}\left(\oplus V_{i}\right)$ in $\Gamma L(d, q)$ of the corresponding decomposition of $V$, since this stabilizer is a maximal $\mathcal{C}_{2}$-subgroup.

Let $h \in H$. For each $v \in V_{i} \backslash\{0\}$, we have $v \in \Delta$ and hence $v^{h} \in \Delta$. Thus $v^{h} \in V_{j}$ for some $j$. We claim that $V_{i}^{h}=V_{j}$. Let $w \in V_{i} \backslash\{0, v\}$. Then $v-w \in V_{i} \backslash\{0\}$ and so $w^{h} \in V_{m} \backslash\{0\}$ and $(v-w)^{h} \in V_{l} \backslash\{0\}$ for some $m, l$. Thus $(v-w)^{h}=v^{h}-w^{h} \in\left(V_{j}+V_{m}\right) \cap V_{l}$ and is non-zero. Because the subspace decomposition is a direct sum, we must have $j=m=l$. Thus $w^{h} \in V_{j}$ and since this holds for all $w \in V_{i}, h$ maps $V_{i}$ to $V_{j}$. It follows that $h \in \operatorname{Stab}_{\Gamma \mathrm{L}}\left(\oplus V_{i}\right)$.

\subsection{The Case $H \in \mathcal{C}_{3}$}

First we describe the maximal $\mathcal{C}_{3}$-subgroups of $\Gamma \mathrm{L}(d, q)$. For each divisor $b>1$ of $d$, write $d=a b$, let $F=F_{q^{b}}$ be an extension field of $F_{q}$ of degree $b$, and identify $V$ with an $a$-dimensional vector space $V\left(a, q^{b}\right)$ over $F$. The stabilizer in $\Gamma L(d, q)$ of this $F$-space structure on $V$ is $\Gamma \mathrm{L}\left(a, q^{b}\right)$. Every maximal $\mathcal{C}_{3}$-subgroup is conjugate to such a subgroup for some $b$. Since $\Gamma \mathrm{L}\left(a, q^{b}\right)$ is transitive on $\Omega$, its 1-closure is $\operatorname{Sym}(\Omega)$, so we will consider 2-closures instead. If $b=d$ let $\xi$ be a primitive element of $F=F_{q^{d}}$, and define $\Delta_{1, d}$ as the 2-relation of (3.1.1) with $q$ replaced by $q^{d}$, that is,

$$
\Delta_{1, d}=\left\{\left(x, x \xi^{p^{i}}\right) \mid x \in \Omega, 0 \leq i<d f\right\}
$$

while if $b<d$, choose an identification of $V$ with $V\left(a, q^{b}\right)$ and define

$$
\Delta_{a, b}=\left\{(\lambda v, v) \mid v \in \Omega, \lambda \in F_{q^{b}}\right\} \quad(\text { for } a=d / b \geq 2) .
$$

Define

$$
k_{3}=2 \quad \text { and } \quad \operatorname{Rel}(3,2)=\left\{\left(\Delta_{a, b}\right)^{g} \mid g \in \mathrm{GL}(d, q), d=a b, b>1\right\}
$$

Proposition 4.3.1. $H \in \mathcal{C}_{3}$ if and only if $\operatorname{Rel}(H, 2) \cap \operatorname{Rel}(3,2) \neq \emptyset$.

Remark 4.3.2. The proof uses a modification of [16, Proposition 84.1]. Suppose that $b<d$, and consider a function $h: V \rightarrow V$, with $V$ identified with the vector space $V\left(a, q^{b}\right)$ over $F$. Then [16, Proposition 84.1] proves that $h \in \Gamma \mathrm{L}\left(a, q^{b}\right)$ if and only if $h$ has the following three properties:

1. $h$ is an automorphism of the additive group of $V$;

2. $h$ sends one-dimensional $F$-subspaces to one-dimensional $F$-subspaces; 
3. if $u$ and $v$ are $F$-linearly independent vectors of $V$, then also their images $u^{h}$ and $v^{h}$ under $h$ are $F$-linearly independent.

Now properties 1 and 2 together imply property 3, and moreover, if we are given that $h \in \Gamma \mathrm{L}(d, q)$, then property 1 holds. Thus for $h \in \Gamma \mathrm{L}(d, q)$, we conclude that $h \in \Gamma \mathrm{L}\left(a, q^{b}\right)$ if and only if property 2 holds.

Proof of Proposition 4.3.1.

It follows from the definition of $\operatorname{Rel}(3,2)$ that each $\mathcal{C}_{i}$-subgroup leaves invariant some relation in $\operatorname{Rel}(3,2)$. Conversely assume that $\operatorname{Rel}(H, 2) \cap \operatorname{Rel}(3,2)$ contains a relation $\Delta$. We must prove that $H \in \mathcal{C}_{3}$. By definition, $\Delta=\Delta_{a, b}^{g}$ for some $g \in \operatorname{GL}(d, q)$ and some factorisation $d=a b$ with $b>1$. Since $\mathcal{C}_{3}$ is closed under conjugacy, we may assume that $\Delta=\Delta_{a, b}$. If $b=d$ then $\Delta$ is as in (3.1.1), and it follows from Proposition 3.1.1 that $H \leq \Gamma \mathrm{L}\left(1, q^{d}\right)$ and hence $H \in \mathcal{C}_{3}$ in this case. So we may assume that $b<d$.

Let $h \in H, F=F_{q^{b}}$. Then for $v \in V\left(a, q^{b}\right)$ and $\lambda \in F,(\lambda v, v) \in \Delta_{a, b}$ and hence $(\lambda v, v)^{h} \in \Delta_{a, b}$. Thus

$$
(\lambda v, v)^{h}=(\mu w, w) \text { for some } \mu \in F \text { and } w \in \Omega .
$$

This implies that $(\lambda v)^{h}=\mu w=\mu v^{h}$. Letting $\lambda$ vary over $F$ we conclude that the $F$ subspace image $\left(\operatorname{Span}_{F}\langle v\rangle\right)^{h}=\operatorname{Span}_{F}\langle w\rangle$. Therefore $h$ has property 2 of Remark 4.3.2, and so $h \in \Gamma \mathrm{L}\left(a, q^{b}\right)$. It follows that $H \leq \Gamma \mathrm{L}\left(a, q^{b}\right)$ and hence $H \in \mathcal{C}_{3}$.

\subsection{The Cases $H \in \mathcal{C}_{4}$ and $H \in \mathcal{C}_{7}$}

The maximal subgroups of $\Gamma \mathrm{L}(V)$ in these two families are stabilizers of tensor decompositions of $V$. The main result of this subsection is Proposition 4.4.1.

For $1 \leq i \leq t$ and $t \geq 2$, let $V_{i}$ be an $n_{i}$-dimensional vector space over the finite field $F_{q}$, such that $V=V_{1} \otimes \ldots \otimes V_{t}$. Then $V$ has dimension $n=\prod_{i=1}^{t} n_{i}$. For each $i$, let $\left\{x_{i j} \mid 1 \leq j \leq n_{i}\right\}$ be a basis of $V_{i}$. Then $B:=\left\{x_{1 j_{1}} \otimes \ldots \otimes x_{t j_{t}} \mid 1 \leq j_{i} \leq n_{i}\right.$ for $\left.1 \leq i \leq t\right\}$ is the corresponding tensor product basis for $V$. If $v_{i}=\sum_{j=1}^{n_{i}} \lambda_{i j} x_{i j} \in V_{i}$, for each $i$, then we denote by $v_{1} \otimes \ldots \otimes v_{t}$ the vector

$$
v_{1} \otimes \ldots \otimes v_{t}=\sum_{\left(j_{1}, \ldots, j_{t}\right)} \prod_{i=1}^{t} \lambda_{i j_{i}}\left(x_{1 j_{1}} \otimes \ldots \otimes x_{t j_{t}}\right)
$$

of $V$. We call such an element of $V$ a simple vector. Note that in this subsection we do not use the usual convention that the $v_{i}$ form a specified basis of $V$. Also we define the action of $\tau$ on $V$ with respect to the tensor product basis $B$, so that in particular, $\tau$ lies in the stabilizer of the tensor decomposition, and $\tau$ maps simple vectors to simple vectors.

Case $\mathcal{C}_{4}$ : For each expression $d=a b$ with $a>1, b>1$ and $a \neq b$, choose a decomposition for $V$ as above with $t=2, n_{1}=a, n_{2}=b$, and write $U_{a}=V_{1}, W_{b}=V_{2}$. Let $\Delta_{a, b}$ be the corresponding set of non-zero simple vectors. The decomposition stabilizer is

$$
\operatorname{Stab}_{\Gamma \mathrm{L}}\left(U_{a} \otimes W_{b}\right)=\left(\mathrm{GL}\left(U_{a}\right) \otimes \operatorname{GL}\left(W_{b}\right)\right) \rtimes\langle\tau\rangle
$$


and $\Delta_{a, b}$ is a $\operatorname{Stab}_{\Gamma \mathrm{L}}\left(U_{a} \otimes W_{b}\right)$-invariant 1-relation. Define $k_{4}=1$ and

$$
\operatorname{Rel}(4,1)=\left\{\begin{array}{cl}
\left\{\left(\Delta_{a, b}\right)^{g} \mid g \in \mathrm{GL}(d, q), d=a b, a \neq b, a, b \geq 2\right\} & \text { if } d \text { is composite but } \\
& \text { not a square of a } \\
& \text { prime, } \\
\emptyset & \text { otherwise }
\end{array}\right.
$$

Case $\mathcal{C}_{7}$ : For each expression $d=c^{t}$ with $c \geq 2$ and $t \geq 2$, choose a decomposition for $V$ as above with $n_{1}=\cdots=n_{t}=c$, and let $\Delta_{c, t}$ be the corresponding set of non-zero simple vectors. We view each $V_{i}$ as a copy of a single $c$-dimensional space $W_{c}$ and write the decomposition as $V=\otimes W_{c}$. The stabilizer is

$$
\operatorname{Stab}_{\Gamma \mathrm{L}}\left(\otimes W_{c}\right)=\left(\mathrm{GL}\left(W_{c}\right) 2_{\otimes} S_{t}\right) \rtimes\langle\tau\rangle,
$$

where

$$
\mathrm{GL}\left(W_{c}\right) \imath_{\otimes} S_{t}=\left(\mathrm{GL}\left(W_{c}\right) \otimes \cdots \otimes \mathrm{GL}\left(W_{c}\right)\right) \rtimes S_{t}
$$

and $\Delta_{c, t}$ is a $\operatorname{Stab}_{\Gamma \mathrm{L}}\left(\otimes W_{c}\right)$-invariant 1-relation. Define $k_{7}=1$ and

$$
\operatorname{Rel}(7,1)=\left\{\begin{array}{cl}
\left\{\left(\Delta_{c, t}\right)^{g} \mid g \in \mathrm{GL}(d, q), d=c^{t}, c \geq 2, t \geq 2\right\} & \text { if } d \text { is a proper power } \\
\emptyset & \text { otherwise }
\end{array}\right.
$$

Proposition 4.4.1. For $i=4$ or $7, H \in \mathcal{C}_{i}$ if and only if $\operatorname{Rel}(H, 1) \cap \operatorname{Rel}(i, 1) \neq \emptyset$.

We derive some properties of simple vectors in tensor decompositions in Subsection 4.4.1, and then prove Proposition 4.4.1 in Subsection 4.4.2.

\subsubsection{Properties of simple vectors}

First we consider addition of simple vectors relative to a tensor decomposition $V=$ $V_{1} \otimes \ldots \otimes V_{t}$ as introduced above. Let $\Delta$ be the set of non-zero simple vectors relative to this decomposition.

Lemma 4.4.2. Let $w_{1}=v_{1} \otimes \ldots \otimes v_{t}$ and $w_{2}=u_{1} \otimes \ldots \otimes u_{t}$ lie in $\Delta$. Then $w_{1}+w_{2} \in \Delta$ if and only if $u_{i}$ is a scalar multiple of $v_{i}$ for all but at most one $i$.

Proof. Suppose $u_{i}$ is a scalar multiple of $v_{i}$ for all but at most one $i$. Without loss of generality we may assume that there exist $\lambda_{2}, \ldots, \lambda_{t} \in F_{q}$ such that $u_{2}=\lambda_{2} v_{2}, \ldots, u_{t}=\lambda_{t} v_{t}$. Set $\lambda=\lambda_{2} \lambda_{3} \ldots \lambda_{t}$. Then $w_{1}+w_{2}=\left(v_{1}+\lambda u_{1}\right) \otimes v_{2} \otimes \ldots \otimes v_{t}$ is simple.

Conversely, suppose $w_{1}+w_{2}$ is simple. If $w_{1}+w_{2}=0$, then $w_{1}=-w_{2}$. This implies that $u_{i}$ is a scalar multiple of $v_{i}$ for all $i$. 
Now suppose that $w_{1}+w_{2} \neq 0$. Let $U_{i}=\operatorname{Span}\left(u_{i}, v_{i}\right)$ for each $i$. Suppose that $\left\{u_{1}, v_{1}\right\}$ and $\left\{u_{2}, v_{2}\right\}$ are linearly independent sets. Note that $w_{1}+w_{2} \in U_{1} \otimes \ldots \otimes U_{t}$. Then since $w_{1}+w_{2}$ is simple, there exist $\lambda_{1}, \lambda_{2}, \lambda_{3}, \lambda_{4} \in F_{q}$ and $e_{i} \in U_{i}$ for $3 \leq i \leq t$ such that

$$
\begin{aligned}
w_{1}+w_{2}= & \left(u_{1} \otimes \ldots \otimes u_{t}\right)+\left(v_{1} \otimes \ldots \otimes v_{t}\right) \\
= & \left(\lambda_{1} u_{1}+\lambda_{2} v_{1}\right) \otimes\left(\lambda_{3} u_{2}+\lambda_{4} v_{2}\right) \otimes e_{3} \otimes \ldots \otimes e_{t} \\
= & \lambda_{1} \lambda_{3}\left(u_{1} \otimes u_{2} \otimes e_{3} \otimes \ldots \otimes e_{t}\right)+\lambda_{1} \lambda_{4}\left(u_{1} \otimes v_{2} \otimes e_{3} \otimes \ldots \otimes e_{t}\right) \\
& +\lambda_{2} \lambda_{3}\left(v_{1} \otimes u_{2} \otimes e_{3} \otimes \ldots \otimes e_{t}\right)+\lambda_{2} \lambda_{4}\left(v_{1} \otimes v_{2} \otimes e_{3} \otimes \ldots \otimes e_{t}\right) .
\end{aligned}
$$

Hence when $t=2$, we have:

$$
u_{1} \otimes u_{2}+v_{1} \otimes v_{2}=\lambda_{1} \lambda_{3}\left(u_{1} \otimes u_{2}\right)+\lambda_{1} \lambda_{4}\left(u_{1} \otimes v_{2}\right)+\lambda_{2} \lambda_{3}\left(v_{1} \otimes u_{2}\right)+\lambda_{2} \lambda_{4}\left(v_{1} \otimes v_{2}\right) .
$$

Since $u_{1} \otimes u_{2}, u_{1} \otimes v_{2}, v_{1} \otimes u_{2}$ and $v_{1} \otimes v_{2}$ are linearly independent, we have $\lambda_{1} \lambda_{3}=\lambda_{2} \lambda_{4}=1$ and $\lambda_{1} \lambda_{4}=\lambda_{2} \lambda_{3}=0$, which is impossible. When $t \geq 3$,

$$
\begin{aligned}
0= & u_{1} \otimes u_{2} \otimes\left(\left(u_{3} \otimes \cdots \otimes u_{t}\right)-\left(\lambda_{1} \lambda_{3} e_{3} \otimes \cdots \otimes e_{t}\right)\right) \\
& +v_{1} \otimes v_{2} \otimes\left(\left(v_{3} \otimes \cdots \otimes v_{t}\right)-\left(\lambda_{2} \lambda_{4} e_{3} \otimes \cdots \otimes e_{t}\right)\right) \\
& -u_{1} \otimes v_{2} \otimes \lambda_{1} \lambda_{4} e_{3} \otimes \cdots \otimes e_{t} \\
& -v_{1} \otimes u_{2} \otimes \lambda_{2} \lambda_{3} e_{3} \otimes \cdots \otimes e_{t}
\end{aligned}
$$

If any of the four summands is non-zero, then it is linearly independent of the sum of the other three summands, and we have a contradiction. Hence each of the summands is 0 . Since $w_{1}, w_{2}, w_{1}+w_{2}$ are all non-zero, it follows that all the $u_{i}, v_{i}, e_{i}$ are non-zero and hence we must have $\lambda_{1} \lambda_{3} \neq 0, \lambda_{2} \lambda_{4} \neq 0$ and $\lambda_{1} \lambda_{4}=\lambda_{2} \lambda_{3}=0$, which is impossible.

Therefore $u_{i}$ is a scalar multiple of $v_{i}$ for all but at most one $i$.

For each $i$, choose $e_{i}$, a non-zero element of $V_{i}$. Define $e:=e_{1} \otimes e_{2} \otimes \ldots \otimes e_{t}$ and

$$
W_{i}:=\left\{e_{1} \otimes \ldots \otimes e_{i-1} \otimes v_{i} \otimes e_{i+1} \otimes \ldots \otimes e_{t} \mid v_{i} \in V_{i}\right\} .
$$

Lemma 4.4.3. With the notation as above, let $g \in \mathrm{GL}(V)$ be a linear transformation such that $e^{g}=e$ and for any simple $w \in V, w^{g}$ is also simple. Then for each $i=1, \ldots, t$, there exists $j$, such that $1 \leq j \leq t$ and $W_{i}^{g} \subseteq W_{j}$.

Proof. Without loss of generality, we may assume that $i=1$. If $\operatorname{dim} V_{1}=1$, then $W_{1}=\langle e\rangle$ and $W_{1}^{g}=W_{1}$, so the result holds with $j=1$. Thus we may assume that $\operatorname{dim} V_{1} \geq 2$. Let $v \in V_{1} \backslash\left\langle e_{1}\right\rangle$. Since $g$ preserves the set of simple vectors,

$$
\left(v \otimes e_{2} \otimes \ldots \otimes e_{t}\right)^{g}=u_{1} \otimes \ldots \otimes u_{t}
$$

for some $u_{i} \in V_{i}, 1 \leq i \leq t$. Since $\left(e_{1} \otimes e_{2} \otimes \ldots \otimes e_{t}\right)+\left(v \otimes e_{2} \otimes \ldots \otimes e_{t}\right)$ is simple, its image $\left(e_{1} \otimes \ldots \otimes e_{t}\right)+\left(u_{1} \otimes \ldots \otimes u_{t}\right)$ under $g$ is also simple. By Lemma 4.4.2, $u_{i}$ is a scalar multiple of $e_{i}$ for all but at most one $i$. Moreover, since $e_{1} \otimes \ldots \otimes e_{t}$ and $v \otimes e_{2} \otimes \ldots \otimes e_{t}$ are 
linearly independent, $e_{1} \otimes \ldots \otimes e_{t}$ and $u_{1} \otimes \ldots \otimes u_{t}$ are linearly independent. Thus there exists precisely one $j$ such that $u_{j} \notin\left\langle e_{j}\right\rangle$. If $v^{\prime} \in V_{1} \backslash\left\langle e_{1}\right\rangle$ and

$$
\left(v^{\prime} \otimes e_{2} \otimes \ldots \otimes e_{t}\right)^{g}=u_{1}^{\prime} \otimes \ldots \otimes u_{t}^{\prime},
$$

then the same argument gives that $u_{i}^{\prime}$ is a scalar multiple of $e_{i}$ for all but one $i$, say $u_{l}^{\prime} \notin\left\langle e_{l}\right\rangle$. Using the fact that $\left(v \otimes e_{2} \otimes \ldots \otimes e_{t}\right)+\left(v^{\prime} \otimes e_{2} \otimes \ldots \otimes e_{t}\right)$ is simple, we deduce that $u_{i}^{\prime}$ is a scalar multiple of $u_{i}$ for all but one $i$. However, if $j \neq l$, then this means that $u_{j}^{\prime} \in\left\langle e_{j}\right\rangle \cap\left\langle u_{j}\right\rangle=\{0\}$ which is not the case. Hence $l=j$, and so $u_{i}^{\prime} \in\left\langle e_{i}\right\rangle$ for all $i \neq j$. Thus

$$
\left(v^{\prime} \otimes e_{2} \otimes \ldots \otimes e_{t}\right)^{g} \in W_{j}
$$

for each $v^{\prime} \in V_{1} \backslash\left\langle e_{1}\right\rangle$. Since also $e^{g}=e \in W_{j}$, it follows that $W_{1}^{g} \subseteq W_{j}$.

Lemma 4.4.4. Let $g \in \mathrm{GL}(V)$ such that $g$ leaves invariant the set of simple vectors, and $g$ fixes each $W_{i}$ pointwise. Then $g=1$.

Proof. We claim that for any simple $w \in V, w^{g}$ is a scalar multiple of $w$. Let $w=$ $v_{1} \otimes v_{2} \otimes \ldots \otimes v_{t}$, and let $l$ be the number of $i$ such that $v_{i} \notin\left\langle e_{i}\right\rangle$. We prove the claim by induction on $l$. By assumption, for $l=0$ and $l=1, w^{g}=w$. Now assume inductively that the claim is true for $l=m$ where $1 \leq m<t$. We will show that it is true for $l=m+1$.

Without loss of generality, we may suppose that

$$
w=v_{1} \otimes \ldots \otimes v_{m+1} \otimes e_{m+2} \ldots \otimes e_{t}
$$

where for $i=1, \ldots, m+1, v_{i} \notin\left\langle e_{i}\right\rangle$. Let

$$
w^{g}=u_{1} \otimes \ldots \otimes u_{t} .
$$

Set

$$
w_{1}=e_{1} \otimes v_{2} \otimes \ldots \otimes v_{m+1} \otimes e_{m+2} \ldots \otimes e_{t}
$$

and

$$
w_{2}=v_{1} \otimes \ldots \otimes v_{m} \otimes e_{m+1} \otimes e_{m+2} \ldots \otimes e_{t} .
$$

Then $w_{1}+w$ and $w_{2}+w$ are simple and hence $\left(w_{1}+w\right)^{g}$ and $\left(w_{2}+w\right)^{g}$ are simple. Also, by induction, $w_{1}^{g}=\lambda_{1} w_{1}$ and $w_{2}^{g}=\lambda_{2} w_{2}$ for some $\lambda_{1}, \lambda_{2} \in F_{q}$.

Thus $\left(w_{1}+w\right)^{g}=\lambda_{1} w_{1}+w^{g}$, and this is a simple vector. So by Lemma 4.4.2, $u_{i}$ is a scalar multiple of the $i$ th component of $w_{1}$ for all but one $i$. Likewise, $u_{i}$ is a scalar multiple of the $i$ th component of $w_{2}$ for all but one $i$. However, $u_{1}$ cannot be a scalar multiple of both $e_{1}$ and $v_{1}$, and $u_{m+1}$ cannot be a scalar multiple of both $e_{m+1}$ and $v_{m+1}$. Therefore for all $i \notin\{1, m+1\}, u_{i}$ is a scalar multiple of the $i$ th component of $w$. Thus $w^{g} \in\langle x\rangle$, where $x=u_{1} \otimes v_{2} \otimes \ldots \otimes v_{m} \otimes u_{m+1} \otimes e_{m+2} \otimes \ldots \otimes e_{t}$. Also, (i) either $u_{1} \in\left\langle e_{1}\right\rangle$ or $u_{m+1} \in\left\langle v_{m+1}\right\rangle$, and (ii) either $u_{1} \in\left\langle v_{1}\right\rangle$ or $u_{m+1} \in\left\langle e_{m+1}\right\rangle$. Since $\left\{e_{1}, v_{1}\right\}$ and $\left\{e_{m+1}, v_{m+1}\right\}$ are both linearly independent sets, we conclude that $\left(\left\langle u_{1}\right\rangle,\left\langle u_{m+1}\right\rangle\right)=\left(\left\langle e_{1}\right\rangle,\left\langle e_{m+1}\right\rangle\right)$ or $\left(\left\langle v_{1}\right\rangle,\left\langle v_{m+1}\right\rangle\right)$. In the former case, by induction, $x^{g} \in\langle x\rangle$, and hence both $x^{g}$ and $w^{g}$ lie in $\langle x\rangle$, contradicting 
the fact that $x$ and $w$ are linearly independent. Hence $\left(\left\langle u_{1}\right\rangle,\left\langle u_{m+1}\right\rangle\right)=\left(\left\langle v_{1}\right\rangle,\left\langle v_{m+1}\right\rangle\right)$, and so $w^{g}$ is a scalar multiple of $w$ and the claim is proved by induction.

Now, using induction on $l$ once again (with $l$ defined as above), we show that $w^{g}=w$ for every simple $w \in W$, and hence that $g=1$. The case $l \leq 1$ is true by assumption. Now assume that this is true for $l=m$ where $1 \leq m<t$ and, without loss of generality, consider $w=v_{1} \otimes \ldots \otimes v_{m+1} \otimes e_{m+2} \ldots \otimes e_{t}$ where $v_{i} \notin\left\langle e_{i}\right\rangle$ for $i=1, \ldots, m+1$. Once again, set $w_{1}=e_{1} \otimes v_{2} \otimes \ldots \otimes v_{m+1} \otimes e_{m+2} \ldots \otimes e_{t}$. Then both $w$ and $w+w_{1}$ are simple. Hence there exist $\lambda, \mu \in F_{q}^{*}$ such that $w^{g}=\lambda w$ and $\left(w+w_{1}\right)^{g}=\mu\left(w+w_{1}\right)$. Also, by the inductive hypothesis, $\left(w_{1}\right)^{g}=w_{1}$. But then $\mu\left(w+w_{1}\right)=\left(w+w_{1}\right)^{g}=w^{g}+w_{1}^{g}=\lambda w+w_{1}$. Since $w$ and $w_{1}$ are linearly independent, $\mu=\lambda=1$ and $w^{g}=w$.

\subsubsection{Proofs for $\mathcal{C}_{4}$ and $\mathcal{C}_{7}$}

Before proving Proposition 4.4.1, we prove the next lemma that makes explicit the important role of simple vectors.

Lemma 4.4.5. With the above notation, let $g \in \Gamma \mathrm{L}(V)=\mathrm{GL}(V) \rtimes\langle\tau\rangle$.

(1) Suppose $V=U \otimes W$, with $\operatorname{dim} U \geq 2, \operatorname{dim} W \geq 2, \operatorname{dim} U \neq \operatorname{dim} W$. If $g$ leaves invariant the set of simple vectors, then $g \in \operatorname{Stab}_{\Gamma L}(U \otimes W)$.

(2) Suppose $V=V_{1} \otimes \ldots \otimes V_{t}$ is the tensor product of $t \geq 2$ copies $V_{1}, \ldots, V_{t}$ of a vector space $W$. If $g$ leaves invariant the set of simple vectors, then $g \in \operatorname{Stab}_{\Gamma \mathrm{L}}\left(\otimes V_{i}\right)$.

Proof. (1) By suitable choice of bases for $U, W$ we may assume that $\tau \in \operatorname{Stab}_{\Gamma L}(U \otimes W)$, as in (4.4.1), and hence that $\tau$ maps simple vectors to simple vectors. Thus replacing $g$ by $g \tau^{i}$ for some $i$, we may assume that $g \in \mathrm{GL}(V)$.

Let $e_{1} \in U, e_{2} \in W$ be any non-zero elements of $U$ and $W$. Replacing $g$ by $g h_{1}$ for an appropriate $h_{1} \in \mathrm{GL}(U) \otimes \mathrm{GL}(W)$ we may assume further that $\left(e_{1} \otimes e_{2}\right)^{g}=e_{1} \otimes e_{2}$. Since $\operatorname{dim} U \neq \operatorname{dim} W$ and $g \in \mathrm{GL}(V)$, Lemma 4.4.3 implies that $\left(e_{1} \otimes W\right)^{g}=e_{1} \otimes W$ and $\left(U \otimes e_{2}\right)^{g}=U \otimes e_{2}$. Thus $g$ induces linear transformations on $e_{1} \otimes W$ and $U \otimes e_{2}$, so replacing $g$ by $g h_{2}$ for an appropriate $h_{2} \in \mathrm{GL}(U) \otimes \mathrm{GL}(W)$, we may assume in addition that $g$ fixes $e_{1} \otimes w$ and $u \otimes e_{2}$ for all $u \in U, w \in W$. Then by Lemma 4.4.4,g=1. Thus we deduce that our original element $g$ was in $\operatorname{Stab}_{\Gamma \mathrm{L}}(U \otimes W)$.

(2) Again by suitable choice of bases for the $V_{i}$ we may assume that $\tau \in \operatorname{Stab}_{\Gamma \mathrm{L}}\left(\otimes V_{i}\right)$, as in (4.4.3), and hence that $\tau$ maps simple vectors to simple vectors. Thus we may replace $g$ by $g \tau^{i}$ for some $i$ and assume that $g \in \mathrm{GL}(V)$.

Let $e_{1}, \ldots, e_{t}$ be any non-zero vectors of $W$. Replacing $g$ by $g h_{1}$ for an appropriate $h_{1} \in \mathrm{GL}(W) \otimes \ldots \otimes \mathrm{GL}(W)$ we may assume that $\left(e_{1} \otimes \ldots \otimes e_{t}\right)^{g}=e_{1} \otimes \ldots \otimes e_{t}$. By Lemma 4.4.3, we then have that, for each $i=1, \ldots, t$, there exists $j_{i}$ such that $1 \leq j_{i} \leq t$ and $\left(e_{1} \otimes \ldots \otimes e_{i-1} \otimes V_{i} \otimes e_{i+1} \otimes \ldots \otimes e_{t}\right)^{g} \subseteq e_{1} \otimes \ldots \otimes V_{j_{i}} \otimes \ldots \otimes e_{t}$. Since $g: V \rightarrow V$ is bijective, the map $i \rightarrow j_{i}$ defines an element of $S_{t}$.

Thus we may further replace the above $g$ by $g h_{2}$ for an appropriate $h_{2} \in \mathrm{GL}(W) \eta_{\otimes} S_{t}$, and assume that $g$ fixes $e_{1} \otimes \ldots \otimes e_{i-1} \otimes w \otimes e_{i+1} \otimes \ldots \otimes e_{t}$ for every $w \in V_{i}$ and every $i$ with $1 \leq i \leq t$. Then an application of Lemma 4.4 .4 concludes the proof.

Now we are ready to prove Proposition 4.4.1. 
Proof of Proposition 4.4.1: Note that the same arguments apply to the case $\mathcal{C}_{7}$, so we only give details of the proof for the case $\mathcal{C}_{4}$. If $H$ is a $\mathcal{C}_{4}$-subgroup then, by definition, $H$ preserves some relation in $\operatorname{Rel}(4,1)$. Conversely suppose that $H$ leaves invariant a relation $\Delta=\left(\Delta_{a, b}\right)^{g}$ in $\operatorname{Rel}(4,1)$, for some $g \in \operatorname{GL}(d, q)$. Since $\mathcal{C}_{4}$ is closed under conjugacy we may assume that $\Delta=\Delta_{a, b}$. By Lemma 4.4.5, $H \leq \operatorname{Stab}_{\Gamma \mathrm{L}}\left(U_{a} \otimes W_{b}\right)$, and hence we conclude that $H \in \mathcal{C}_{4}$

\subsection{The Case $H \in \mathcal{C}_{5}$}

First we describe the maximal $\mathcal{C}_{5}$-subgroups of $\Gamma \mathrm{L}(d, q)$. Recall that $q=p^{f}$, that $Z$ is the subgroup of scalars, and that $\left\{v_{1}, \ldots, v_{d}\right\}$ is a specified basis for $V$. For a divisor $a$ of $f$ with $a<f$ let $q_{0}=p^{a}$, let $F_{q_{0}}$ denote the proper subfield of $F_{q}$ of order $q_{0}$, and let $V_{0}=\operatorname{Span}_{F_{q_{0}}}\left\langle v_{1}, \ldots, v_{d}\right\rangle$. Then the stabilizer $\operatorname{Stab}_{\Gamma \mathrm{L}}\left(F_{q} V_{0}\right)$ of $F_{q} V_{0}=\left\{\lambda v \mid v \in V_{0}, \lambda \in F_{q}\right\}$ in $\Gamma \mathrm{L}(d, q)$ is a maximal $\mathcal{C}_{5}$-subgroup. We describe its structure below. Let

$$
\Delta_{a}=\left\{\lambda u \mid \lambda \in F_{q}^{*}, u \in V_{0} \backslash\{0\}\right\}=F_{q} V_{0} \backslash\{0\}
$$

and define

$$
k_{5}=1 \quad \text { and } \quad \operatorname{Rel}(5,1)=\left\{\begin{array}{cl}
\left\{\left(\Delta_{a}\right)^{g}|g \in \mathrm{GL}(d, q), a| f, a<f\right\} & \text { if } f>1 \\
\emptyset & \text { if } f=1
\end{array}\right.
$$

Proposition 4.5.1. $H \in \mathcal{C}_{5}$ if and only if $\operatorname{Rel}(H, 1) \cap \operatorname{Rel}(5,1) \neq \emptyset$.

We will see that this result follows from Proposition 4.4.1. Using the notation of Subsection 4.4, identify $V$ with the vector space $V_{0} \otimes F_{q}=V_{0} \otimes_{F_{q_{0}}} F_{q}$ of dimension $d f / a$ over $F_{q_{0}}$, regarding $F_{q}$ as a vector space of dimension $f / a$ over $F_{q_{0}}$, see [11, Section 4.5]. Then $V_{0}$ is identified with the subset $\left\{u \otimes 1 \mid u \in V_{0}\right\}$ of $V \otimes F_{q}$. The corresponding maximal $\mathcal{C}_{5}$-subgroup is

$$
\operatorname{Stab}_{\Gamma \mathrm{L}}\left(F_{q} V_{0}\right)=\Gamma \mathrm{L}(d, q) \cap \operatorname{Stab}_{\Gamma \mathrm{L}\left(d f / a, q_{0}\right)}\left(V_{0} \otimes F_{q}\right)=\left(\mathrm{GL}\left(d, q_{0}\right) \circ Z\right) \rtimes\langle\tau\rangle,
$$

the stabilizer in $\Gamma \mathrm{L}(d, q)$ of the tensor decomposition $V_{0} \otimes_{F_{q_{0}}} F_{q}$ (as distinct from the stabilizer in $\Gamma \mathrm{L}\left(d f / a, q_{0}\right)$ of $V_{0} \otimes_{F_{q_{0}}} F_{q}$, which as in Subsection 4.4.2 is a maximal $\mathcal{C}_{4^{-}}$ subgroup of $\Gamma \mathrm{L}\left(d f / a, q_{0}\right)$, see (4.4.1)). Under this identification, $F_{q} V_{0}$ is identified with the set of simple vectors in $V_{0} \otimes F_{q}$. Thus, by Proposition 4.4.1 (and its short proof), $H$ preserves $\Delta_{a}$ if and only if $H$ is contained in the subgroup displayed at (4.5.2), which is a maximal $\mathcal{C}_{5}$-subgroup. Now Proposition 4.5.1 follows immediately.

\subsection{The Case $H \in \mathcal{C}_{6}$}

For a prime $r$, an $r$-group $R$ is said to be of symplectic type if every characteristic abelian subgroup of $R$ is cyclic. Each $\mathcal{C}_{6}$-subgroup has, as a normal subgroup, an absolutely irreducible symplectic type $r$-group of exponent $r \operatorname{gcd}(2, r)$, for some $r \neq p$, and the maximal $\mathcal{C}_{6}$-subgroups are the normalizers of such $r$-groups in $\Gamma \mathrm{L}(d, q)$. 
Let $R$ be such an $r$-subgroup of $\Gamma L(d, q)$. We refer to [11, Sections 4.6 and 7.6] for much of the information in this subsection. By [11, Proposition 4.6.3], $R \leq \mathrm{GL}(d, q)$ and the representation of $R$ on $V$ can be realised over the subfield $F_{p^{e}}$ of $F_{q}$, where $e$ is the least positive integer such that $p^{e} \equiv 1(\bmod |Z(R)|)$. Replacing $R$ by a conjugate in $\Gamma \mathrm{L}(d, q)$ we may assume that $R \leq \mathrm{GL}\left(V_{0}\right)$, where $V_{0}=\operatorname{Span}_{F_{p} e}\left\langle v_{1}, \ldots, v_{d}\right\rangle$ and $\left\{v_{1}, \ldots, v_{d}\right\}$ is the basis introduced in Subsection 2.1. Choose a set $\mathcal{R}$ of representatives of the $\Gamma \mathrm{L}(d, q)$ conjugacy classes of these subgroups $R$ such that each subgroup is contained in $\operatorname{GL}\left(V_{0}\right)$. By [1, Theorem BГ] (or see [11, Theorem 3.1.1 and Table 4.6A]), $|\mathcal{R}|=1$ if $r$ is odd, and is 3 if $r=2$. For each $R \in \mathcal{R}$, define a 2-relation $\Delta_{R}$ by

$$
\Delta_{R}=\left\{(v, w) \mid v, w \in F_{q} V_{0}, v^{R}=w^{R}\right\}
$$

let $k_{6}=2$, and define

$$
\operatorname{Rel}(6,2)=\left\{\begin{array}{cl}
\left\{\left(\Delta_{R}\right)^{g} \mid g \in \Gamma \mathrm{L}(d, q), R \in \mathcal{R}\right\} & \text { if } d \text { is a prime power } \\
\emptyset & \text { otherwise }
\end{array}\right.
$$

Proposition 4.6.1. $H \in \mathcal{C}_{6}$ if and only if $\operatorname{Rel}(H, 2) \cap \operatorname{Rel}(6,2) \neq \emptyset$.

\subsubsection{Structure of the groups $R$ and their normalisers}

By $\left[1,3.15\right.$ and Theorem A.4], the normalizer $M:=N_{\Gamma \mathrm{L}(d, q)}(R)$ leaves $F_{q} V_{0}$ invariant and, identifying $V$ with $V_{0} \otimes_{F_{p}} F_{q}$ (as discussed in Subsection 4.5), $M$ is given by

$$
M=N_{\Gamma \mathrm{L}(d, q)}(R)=\left(Z \circ M_{0}\right) \cdot\langle\tau\rangle \quad \text { where } \quad M_{0}=N_{\mathrm{GL}\left(V_{0}\right)}(R) .
$$

The possible structures for the groups $R \in \mathcal{R}$, and the corresponding subgroups $M_{0}=$ $Z_{p^{e}-1} \circ M_{1}$, are summarized in Table 3 , see [11, Tables 4.6A and 4.6.B], where $R_{0}$ denotes the group

$$
R_{0}=\left\langle x, y, z \mid x^{r}=y^{r}=z^{r}=[x, z]=[y, z]=1,[y, x]=z\right\rangle
$$

and $m$ is such that $d=r^{m}$ and $m \geq 1$ for all types. Note that no \pm sign appears in the notation $2^{1+2 m}$ for Type 4 since $Z_{4} \circ 2_{+}^{1+2 m} \cong Z_{4} \circ 2_{-}^{1+2 m}$.

A crucial link between the definition of $\mathcal{C}_{6}$-subgroups and the relation set $\operatorname{Rel}(6,2)$ is explored in the next lemma. Note that, by its definition, $\Delta_{R}=\bigcup_{\Sigma} \Sigma \times \Sigma$ where the union is over all $R$-orbits $\Sigma \subseteq F_{q} V_{0}$.

Lemma 4.6.2. Let $g \in \Gamma \mathrm{L}(d, q), R \in \mathcal{R}$, and let $\Delta_{R}, M=N_{\Gamma \mathrm{L}(d, q)}(R), M_{0}$ be as in (4.6.1), (4.6.3). Then

(a) $\Delta_{R}$ is $M$-invariant;

(b) $g$ leaves $\Delta_{R}$ invariant if and only if $g=\lambda h \tau^{i} \in\left(Z \circ \operatorname{GL}\left(V_{0}\right)\right)\langle\tau\rangle$, where $\lambda \in Z$ and $h \in \mathrm{GL}\left(V_{0}\right)$ such that $h$ leaves $\Delta_{R}$ invariant;

(c) if $h \in \mathrm{GL}\left(V_{0}\right)$ and $h$ leaves $\Delta_{R}$ invariant, then $h$ permutes amongst themselves the $R$-orbits in $V_{0}$. 


\begin{tabular}{c|cccccc}
\hline Type & $r$ & $e$ & $\begin{array}{c}\text { Structure } \\
\text { of } R\end{array}$ & $|Z(R)| \begin{array}{c}\text { Notation } \\
\text { for } R\end{array}$ & $\begin{array}{c}\text { Structure } \\
\text { of } M_{0}\end{array}$ \\
\hline 1 & odd & $e$ & $\overbrace{R_{0} \circ \cdots \circ R_{0}}^{m}$ & $r$ & $r^{1+2 m}$ & $R \cdot \operatorname{Sp}(2 m, r)$ \\
2 & 2 & 1 & $\overbrace{D_{8} \circ \cdots \circ D_{8}}^{m m}$ & 2 & $2_{+}{ }^{1+2 m}$ & $R \cdot \mathrm{O}_{2 m}^{+}(2)$ \\
3 & 2 & 1 & $\overbrace{D_{8} \circ \cdots \circ D_{8} \circ Q_{8}}^{m-1}$ & 2 & $2_{-}{ }^{1+2 m}$ & $R \cdot \mathrm{O}_{2 m}^{-}(2)$ \\
4 & 2 & 1 or 2 & $Z_{4} \circ \overbrace{D_{8} \circ \cdots \circ D_{8}}^{m}$ & 4 & $4 \circ 2^{1+2 m}$ & $R \cdot \operatorname{Sp}(2 m, 2)$ \\
\hline
\end{tabular}

Table 3: Posssible structures for $R$ and $M_{0}$

Proof. (a) Let $\Sigma=v^{R} \subseteq F_{q} V_{0}$. Since $M$ leaves $F_{q} V_{0}$ invariant and $R \unlhd M$, for each $h \in M$ we have $v^{h} \in F_{q} V_{0}$, and $\Sigma^{h}=\left(v^{R}\right)^{h}=\left(v^{h}\right)^{R}$. Hence $\Sigma^{h}$ is also an $R$-orbit in $F_{q} V_{0}$, and it follows from the description of $\Delta_{R}$ above that $h$ leaves $\Delta_{R}$ invariant.

(b) Suppose that $g$ leaves $\Delta_{R}$ invariant. Then by the definition of $\Delta_{R}, g$ leaves the set $F_{q} V_{0}$ invariant. By Proposition 4.5.1, $g \in \operatorname{Stab}_{\Gamma \mathrm{L}}\left(F_{q} V_{0}\right)=\left(Z \circ \mathrm{GL}\left(V_{0}\right)\right) \rtimes\langle\tau\rangle$. Thus $g=\lambda h \tau^{i}$ for some $i$, with $\lambda \in Z$ and $h \in \mathrm{GL}\left(V_{0}\right)$. Now $\lambda, \tau^{i} \in M$ by (4.6.3), and hence by part (a), $\lambda$ and $\tau^{i}$ leave $\Delta_{R}$ invariant. Therefore also $h$ leaves $\Delta_{R}$ invariant.

Conversely, suppose that $g=\lambda h \tau^{i} \in\left(\mathrm{GL}\left(V_{0}\right) \circ Z\right) \rtimes\langle\tau\rangle$ with $\lambda \in Z, h \in \mathrm{GL}\left(V_{0}\right)$, and $h$ leaves $\Delta_{R}$ invariant. By (a), $\lambda$ and $\tau^{i}$ both leave $\Delta_{R}$ invariant, and hence so also does g.

(c) Finally suppose that $h \in \mathrm{GL}\left(V_{0}\right)$ and $h$ leaves $\Delta_{R}$ invariant. Let $\Sigma=v^{R} \subseteq V_{0}$, and consider an arbitrary $w \in \Sigma$. Then $(v, w) \in \Sigma \times \Sigma \subseteq \Delta_{R}$ and so, by assumption, also $(v, w)^{h}=\left(v^{h}, w^{h}\right) \in \Delta_{R}$. As we noted above, this means that $\left(v^{h}, w^{h}\right) \in \Sigma^{\prime} \times \Sigma^{\prime}$ for some $R$-orbit $\Sigma^{\prime}$ in $F_{q} V_{0}$, and in fact $\Sigma^{\prime} \subseteq V_{0}$ since $h \in \mathrm{GL}\left(V_{0}\right)$. Hence $\Sigma^{\prime}=\left(v^{h}\right)^{R}$ and $w^{h} \in \Sigma^{\prime}$, and since this holds for arbitrary $w \in \Sigma$ it follows that $\Sigma^{h}=\Sigma^{\prime}$.

We now use this information to partially prove Proposition 4.6.1.

Lemma 4.6.3. Proposition 4.6.1 holds provided the implication (4.6.5) below holds for each $R \in \mathcal{R}$ and its corresponding subgroup $M_{0}$ defined in (4.6.3).

$$
\text { If } h \in \mathrm{GL}\left(V_{0}\right) \text { and } h \text { leaves } \Delta_{R} \text { invariant, then } h \in M_{0} \text {. }
$$

Proof. We assume that (4.6.5) holds and use it to prove Proposition 4.6.1. Suppose first that $H \in \mathcal{C}_{6}$. Then $H$ has as a normal subgroup $R^{g}$ for some $R \in \mathcal{R}$ and $g \in \Gamma L(d, q)$. By Lemma 4.6.2(a), $H$ leaves $\left(\Delta_{R}\right)^{g}$ invariant and hence $\operatorname{Rel}(6,2) \cap \operatorname{Rel}(H, 2) \neq \emptyset$.

Conversely, suppose that $H \leq \Gamma \mathrm{L}(d, q)$ and $\operatorname{Rel}(H, 2) \cap \operatorname{Rel}(6,2)$ contains $\Delta_{R}^{g}$ for some $R \in \mathcal{R}$ and $g \in \Gamma \mathrm{L}(d, q)$. It is sufficient to prove that $H$ normalises $R^{g}$, since this implies that $H \in \mathcal{C}_{6}$. Since both $\mathcal{C}_{6}$ and $\operatorname{Rel}(6,2)$ are closed under conjugacy by elements of $\Gamma \mathrm{L}(d, q)$, we may assume that $g=1$. Let $x \in H$. Then $\Delta_{R}^{x}=\Delta_{R}$. By Lemma 4.6.2(b), $x=\lambda h \tau^{i} \in\left(Z \circ \mathrm{GL}\left(V_{0}\right)\right)\langle\tau\rangle$, where $\lambda \in Z$ and $h \in \mathrm{GL}\left(V_{0}\right)$ such that $\Delta_{R}^{h}=\Delta_{R}$. Now $\lambda$ 
and $\tau^{i}$ normalise $R$, and by our assumption (4.6.5) holds, so also $h$ normalises $R$. Hence $x$ normalises $R$.

Remark 4.6.4. We sketch the strategy that we will use to complete the proof of the implication (4.6.5). Assume that $h \in \mathrm{GL}\left(V_{0}\right)$ and $\left(\Delta_{R}\right)^{h}=\Delta_{R}$, and $M_{0}=N_{\mathrm{GL}\left(V_{0}\right)}(R)$ so that $M_{0}$ contains the scalars $Z_{p^{e}-1}$ of $\mathrm{GL}\left(V_{0}\right)$. We must prove that $h \in M_{0}$ or equivalently, setting $H:=\left\langle M_{0}, h\right\rangle$, that $H=M_{0}$. By Lemma 4.6.2(c), it follows that $H$ permutes amongst themselves the $R$-orbits in $V_{0}$. We will obtain information about these $R$-orbits and argue that no proper overgroup of $M_{0}$ in $\mathrm{GL}\left(V_{0}\right)$ can permute the $R$-orbits in $V_{0}$. For a subgroup $L \leq \mathrm{GL}\left(d, p^{e}\right)$, we write $\bar{L}$ for $L Z_{p^{e}-1} / Z_{p^{e}-1} \leq \operatorname{PGL}\left(d, p^{e}\right)$. Some of our arguments concern overgroups of $\bar{M}_{0}$ in $\operatorname{PGL}\left(d, p^{e}\right)$.

\subsubsection{Proof of (4.6.5) for $d=2$, and for Type 2 with $d=4$}

Suppose first that $d=r=2$. Then $p$ is odd and, by Table 3 , there are three groups $R$ to consider. Let $H$ be as in Remark 4.6.4. If $R=D_{8}$ or $Q_{8}$ then $e=1$ and we give explicit generators for these groups $R$ in terms of the following matrices

$$
a=\left(\begin{array}{cc}
\lambda & \mu \\
\mu & -\lambda
\end{array}\right), \quad b=\left(\begin{array}{cc}
0 & 1 \\
-1 & 0
\end{array}\right) \quad \text { and } \quad c=\left(\begin{array}{cc}
1 & 0 \\
0 & -1
\end{array}\right)
$$

where $\lambda, \mu \in F_{p}$ such that $\lambda^{2}+\mu^{2}=-1$. The group $Q_{8}=\langle a, b\rangle$ (see [11, p.154]) and $D_{8}=\langle b, c\rangle$.

Type 2. $R=D_{8}, e=1$, so $V_{0}=\operatorname{Span}_{F_{p}}\left\langle v_{1}, v_{2}\right\rangle$ and this time $M_{0}=\left(Z_{p-1} \circ R\right) . \mathrm{O}^{+}(2,2)$. We may take $R=\langle b, c\rangle$. There are precisely two, or three pairs of 1 -spaces in $V_{0}$ (according as $p \equiv 3$ or $1(\bmod 4)$ respectively) such that each $R$-orbit containing a vector in one of these 1-spaces consists entirely of vectors from one of the pairs of 1-spaces. These are the pairs $\left\{\operatorname{Span}_{F_{p}}\left\langle v_{1}\right\rangle, \operatorname{Span}_{F_{p}}\left\langle v_{2}\right\rangle\right\}$ and $\left\{\operatorname{Span}_{F_{p}}\left\langle v_{1}+v_{2}\right\rangle, \operatorname{Span}_{F_{p}}\left\langle v_{1}-v_{2}\right\rangle\right\}$, and also, if $p \equiv 1(\bmod 4)$, the pair $\left\{\operatorname{Span}_{F_{p}}\left\langle i v_{1}+v_{2}\right\rangle, \operatorname{Span}_{F_{p}}\left\langle-i v_{1}+v_{2}\right\rangle\right\}$, where $i^{2}=-1$. All other $R$-orbits in $V_{0} \backslash\{0\}$ contain vectors from four 1-spaces in $V_{0}$. Thus $H$ preserves this set of four, respectively six, 1-spaces setwise, permuting them in pairs, and moreover $M_{0}$ induces a transitive action of $D_{8}$ on four of these 1-spaces (and fixes the other two setwise in the case $p \equiv 1(\bmod 4))$. It follows that $H$ acts as $D_{8}$ or $S_{4}$ on these 1-spaces with kernel the scalars $Z\left(\mathrm{GL}\left(V_{0}\right)\right)=Z_{p-1}$ (since the kernel fixes at least four 1-spaces). Thus $H=M_{0}$, or $p \equiv 1(\bmod 4)$ and $|H|=3\left|M_{0}\right|$. Suppose that $H=M_{0} .3$. Then $H=N_{\left.\mathrm{GL}\left(V_{0}\right)\right)}(\langle i I\rangle \circ R)$, that is, the normaliser of a symplectic type 2-group of Type 4 (see Table 3 ). Now

$$
h=\left(\begin{array}{cc}
0 & -1 \\
-i & 0
\end{array}\right)
$$

normalises $\langle i I\rangle \circ R$ and hence belongs to $H$. Moreover, $\left(v_{1}, v_{2}\right) \in \Delta_{R}$. Now $v_{1}^{R}=$ $\left\{v_{1},-v_{1}, v_{2},-v_{2}\right\}$ while $\left(v_{1}, v_{2}\right)^{h}=\left(-v_{2},-i v_{1}\right) \notin \Delta_{R}$. This contradicts the fact that $H$ preserves $\Delta_{R}$. Thus $H=M_{0}$.

Type 3. $R=Q_{8}, e=1$, so $V_{0}=\operatorname{Span}_{F_{p}}\left\langle v_{1}, v_{2}\right\rangle$ and $M_{0}=\left(Z_{p-1} \circ R\right) \cdot \mathrm{O}^{-}(2,2) \cong Z_{p-1} . S_{4}$. If $p^{e}=p=3$ then $M_{0}=\mathrm{GL}\left(V_{0}\right)$ and hence $H=M_{0}$ in this case, so we may assume that 
$p>3$. We claim that $H$ cannot contain $\mathrm{SL}(2, p)$. Each $R$-orbit in $V_{0}$ involves vectors in either two or four 1-spaces (note that $-I \in R$ ). Thus for a non-zero vector $v \in V_{0}$ there exist $u, w \in V_{0}$ such that $u \in v^{R}, w \notin v^{R}, u, v, w$ lie in distinct 1-spaces, and some element of $\operatorname{SL}(2, p)$ maps $(v, u)$ to $(v, w)$. By the definition of $\Delta_{R},(v, u) \in \Delta_{R}$ and $(v, w) \notin \Delta_{R}$, and hence $\operatorname{SL}(2, p)$ does not preserve $\Delta_{R}$. Hence $H$ does not contain $\mathrm{SL}(2, p)$, as claimed. By Dickson's Theorem 2.4.1, the only proper overgroups of $\bar{M}_{0}$ in $\operatorname{PGL}(2, p)$ contain $\operatorname{PSL}(2, p)$, and hence $H=M_{0}$.

Type 4. $R=Z_{4} \circ Q_{8}=Z_{4} \circ D_{8}, e \leq 2$, so $V_{0}=\operatorname{Span}_{F_{p}^{e}}\left\langle v_{1}, v_{2}\right\rangle$ and in particular, $p^{e} \geq 5$. We may take $R$ to be the group generated by the matrices $a, b$ above together with $i I \in Z_{p^{e}-1}$, where $i^{2}=-1$. Here $M_{0}=\left(Z_{p^{e}-1} \circ R\right) \cdot \operatorname{Sp}(2,2) \cong Z_{p^{e}-1} \cdot S_{4}$. As in the Type 3 case, each $R$-orbit in $V_{0}$ involves vectors in either two or four 1 -spaces. Thus for a non-zero vector $v \in V_{0}$ there exist $u, w \in V_{0}$ such that $u \in v^{R}, w \notin v^{R}, u, v, w$ lie in distinct 1-spaces, and some element of $\operatorname{SL}(2, p)$ maps $(v, u)$ to $(v, w)$. By the definition of $\Delta_{R},(v, u) \in \Delta_{R}$ and $(v, w) \notin \Delta_{R}$, and hence SL $(2, p)$ does not preserve $\Delta_{R}$. By Dickson's Theorem 2.4.1, the only proper overgroups of $\bar{M}_{0}$ in $\operatorname{PGL}(2, p)$ contain $\operatorname{PSL}(2, p)$ and hence $H=M_{0}$.

Finally we consider the Type 2 group when $d=4$. We first give a general result concerning Type 2 and Type 3 groups in arbitrary dimension $d=2^{m} \geq 4$.

Lemma 4.6.5. As in Remark 4.6.4, suppose that $M_{0}<H \leq \mathrm{GL}\left(V_{0}\right)$ and that $H$ permutes amongst themselves the $R$-orbits in $V_{0}$. Then $H$ does not lie in $\mathcal{C}_{6}$.

Proof. Suppose that $H$ lies in the class $\mathcal{C}_{6}$ and so is the normaliser of a symplectic type $r$-group $\hat{R}$. Note that $r$ is determined by $d$. Since $H$ does not normalise $R$, it follows that $R$ is of Type 2 or 3 and $\hat{R}=Z_{4} \circ R$ is of Type 4 . Since $H \neq M_{0}$, it follows that $H$ is equal to the normaliser of $\hat{R}$ (since $M_{0}$ is maximal in $\left.N_{\mathrm{GL}\left(V_{0}\right)}(\hat{R})\right)$ ).

Let $S=\langle b, c\rangle$ and $S^{\prime}=\langle a, b\rangle$ with $a, b, c$ as defined at the beginning of this Subsection and let $U$ be a 2-dimensional $F_{p}$-vector space upon which $S$ and $S^{\prime}$ act. Then $V_{0}=$ $U \otimes \cdots \otimes U$ and $R=S \circ \cdots \circ S$ if $R$ is of Type 2 and $R=S \circ \cdots \circ S \circ S^{\prime}$ if $R$ is of Type 3. Let $v_{1}=(1,0), v_{2}=(0,1) \in U$ and let $\Sigma=\left(v_{1} \otimes \cdots \otimes v_{1}\right)^{R}$. Then $\Sigma=\left\{w_{1} \otimes \cdots \otimes w_{m} \mid w_{1}, \ldots, w_{m-1} \in v_{1}^{S}, w_{m} \in \Sigma_{0}\right\}$, where $\Sigma_{0}=v_{1}^{S}$ if $R$ is of Type 2 , or $\Sigma_{0}=v_{1}^{S^{\prime}}$ if $R$ is of Type 3. Let $h \in \mathrm{GL}(2, p)$ as given in (4.6.6), and let $\bar{h}=(h, 1, \ldots, 1) \in$ $\operatorname{GL}(2, p) \circ \cdots \circ \mathrm{GL}(2, p)$. Since $h$ normalises $\langle i I\rangle \circ S$, where $i^{2}=-1$, it follows that $\bar{h}$ normalises $\langle i I\rangle \circ R$. Thus $\bar{h} \in H=N_{\mathrm{GL}\left(V_{0}\right)}(\langle i I\rangle \circ R)$. As seen in the proof for Type 2 with $d=2,\left(v_{1}, v_{2}\right) \in \Delta_{S}$, but $\left(v_{1}, v_{2}\right)^{h} \notin \Delta_{S}$, with $\Delta_{S}$ as in (4.6.1) on $U \times U$. Hence it follows that $\left(v_{1} \otimes \cdots \otimes v_{1}, v_{2} \otimes v_{1} \otimes \cdots \otimes v_{1}\right) \in \Delta_{R}$ while $\left(v_{1} \otimes \cdots \otimes v_{1}, v_{2} \otimes v_{1} \otimes \cdots \otimes v_{1}\right)^{\bar{h}} \notin \Delta_{R}$. Thus $H$ does not preserve $\Delta_{R}$, and hence does not permute the $R$-orbits in $V_{0}$. This contradiction completes the proof.

Type 2 with $d=4$. $R=D_{8} \circ D_{8}=Q_{8} \circ Q_{8}, e=1$, so $V_{0}=\operatorname{Span}_{F_{p}}\left\langle v_{1}, v_{2}, v_{3}, v_{4}\right\rangle$, and $M_{0}=\left(Z_{p-1} \circ R\right) \cdot \mathrm{O}^{+}(4,2)$. Here $M_{0}$ preserves a tensor decomposition $V_{0}=U_{1} \otimes_{F_{p}} U_{2}$, where each $U_{i} \cong F_{p}^{2}$. Thus writing $\bar{M}_{0}=M_{0} / Z_{p-1}$, we have $\bar{M}_{0}=\bar{R} \rtimes O^{+}(4,2)=\left(S_{4} \times S_{4}\right) .2$ contained in $\mathrm{PGO}^{+}(4, p)=(\operatorname{PGL}(2, p) \times \operatorname{PGL}(2, p))$.2. Suppose first that $H$ preserves this 
tensor decomposition. Since $M_{0}$ interchanges the two tensor factors $U_{1}$ and $U_{2}$, so also does $H$. Thus the index 2 subgroup $H_{0}$ of $H$ fixing $U_{1}$ and $U_{2}$ projects to isomorphic subgroups of the two factors $\operatorname{PGL}(2, p)$ in $\mathrm{PGO}^{+}(4, p)$. Since each proper overgroup of $S_{4}$ in $\operatorname{PGL}(2, p)$ contains $\operatorname{PSL}(2, p)$, and since $\bar{H}_{0}$ contains $S_{4} \times S_{4}$, it follows that either $H=M_{0}$, or $\bar{H}_{0}$ contains $\operatorname{PSL}(2, p) \times \operatorname{PSL}(2, p)$. Assume the latter. Then $H_{0}$ is transitive on the $(p+1)^{2} 1$-spaces of simple vectors in $V_{0}$. Now $H_{0}$ must permute $R$-orbits, and it follows from the discussion of Type 2 groups with $d=2$ that $H_{0}$ fixes a subset of $36=6 \times 6$, or $16=4 \times 4$ such 1 -spaces. Hence $p=3$ or 5 . In the former case $\bar{M}_{0}=\mathrm{PGO}^{+}(4, p)$ and hence $H=M_{0}$. Also if $p=5$ then $\operatorname{PSL}(2,5)$ is 2-transitive on the six 1 -spaces of the $U_{i}$, whereas $H_{0}$ preserves a pairing of these 1 -spaces. Thus again in this case we can only have $H=M_{0}$.

Thus we may assume that $H$ does not preserve the tensor decomposition (and in particular does not preserve modulo scalars an orthogonal form of +-type on $V_{0}$ ). Since $e=1$ and since $M_{0}$ is absolutely irreducible on $V_{0}$, it follows (see Subsection 2.2) from Lemma 4.6.5 that the group induced by $H$ on $V_{0}$ does not lie in the class $\mathcal{C}_{i}$ for $i=1$ and $3 \leq i \leq 7$. Also $H$ fixes setwise a subset of 36 or 16 of the 1 -spaces of $V_{0}$, and hence $H$ does not contain $\mathrm{SL}(4, p)$. Thus $H$ lies in $\mathcal{C}_{2} \cup \mathcal{C}_{8} \cup \mathcal{C}_{9}$.

Suppose first that $H$ lies in $\mathcal{C}_{2}$. Then since $M_{0} \leqslant H, H$ must preserve a decomposition $V=W_{1} \oplus W_{2}$ with $\operatorname{dim} W_{i}=2$. Hence $H$ contains a subgroup $K$ of index 2 fixing $W_{1}$ and $W_{2}$ setwise. This means that $K$ is reducible. However $K \cap M_{0}$ must contain $R$, as $\bar{R}$ is the unique minimal normal subgroup of $\bar{M}_{0}$, and the subgroup $R$ is irreducible, implying that that $K$ is irreducible. Therefore $H$ does not lie in $\mathcal{C}_{2}$.

Thus $H$ lies in $\mathcal{C}_{8} \cup \mathcal{C}_{9}$. As we remarked above, $H$ does not preserve modulo scalars a quadratic form of +-type. If $R \leq H \leq \mathrm{GO}^{-}(4, p)$ then, modulo scalars, $\bar{R} \cong Z_{2}^{4} \leq$ $\mathrm{P}^{-}(4, p) \cong \operatorname{PSL}\left(2, p^{2}\right)$, which is impossible. Since $e=1$, the only other possibility is that $H \leqslant \operatorname{GSp}(4, p)$ or $H \in \mathcal{C}_{9}$. Since $\operatorname{Sp}(4, p)$ is transitive on the 1-spaces of $V_{0}$ while $H$ fixes setwise a subset of 36 or 16 of these 1-spaces, it follows that $H$ does not contain $\operatorname{Sp}(4, p)$. Applying Aschbacher's theorem [1] to $H$ (as subgroup of $\operatorname{GL}(4, p)$ or $\operatorname{GSp}(4, p)$ ) we deduce that $\bar{H}$ is almost simple. Using the results of $[6,13]$, we conclude that the simple group involved in $H$ must be among $A_{n}$ for some $n \leq 7, \operatorname{PSL}\left(2, q^{\prime}\right)$ (with $q^{\prime}=p, p^{2}$

or 7$)$, $\operatorname{PSL}(3,4)$, or $\operatorname{PSU}(4,2)$. Since $\bar{H}$ contains $\bar{M}_{0}=\left(S_{4} \times S_{4}\right) .2$, and has an orbit of length 36 or 16 on 1 -spaces, we obtain a contradiction.

\subsubsection{Completion of the proof of (4.6.5)}

As in Remark 4.6.4, suppose that $M_{0}<H \leq \mathrm{GL}\left(V_{0}\right)$ and that $H$ permutes amongst themselves the $R$-orbits in $V_{0}$. We will derive a contradiction. First we find possibilities for proper overgroups of $M_{0}$ in $\mathrm{GL}\left(V_{0}\right)$. By the previous subsection we may assume that $d \geq 3$, and if $d=4$ then $R$ is of Type 3 or 4 .

We observe that, modulo scalars, we have

$$
\overline{M_{0}^{\prime}}= \begin{cases}\bar{R} \cdot \operatorname{Sp}(2 m, r)^{\prime} \quad d=r^{m} \geq 3, & R \text { of Type } 1 \text { or } 4 \\ \bar{R} \cdot \Omega^{+}(2 m, 2), & d=2^{m} \geq 8, \quad R \text { of Type } 2 \\ \bar{R} \cdot \Omega^{-}(2 m, 2), & d=2^{m} \geq 4, \quad R \text { of Type } 3\end{cases}
$$


and that $\bar{R}$ is the unique minimal normal subgroup of $\overline{M_{0}^{\prime}}$. It is important to our proof that $M_{0}^{\prime}$ permutes the $R$-orbits nontrivially and we prove this next.

Lemma 4.6.6. For $d \geq 3$, the group $M_{0}^{\prime}$ acts non-trivially on the set of $R$-orbits.

Proof. Suppose that $M_{0}^{\prime}$ fixes each $R$-orbit setwise. It can be easily seen from the representations of the groups $R$ given in [11, p151-154] that there is an orbit $\Delta$ of $R$ on $V_{0}$ upon which $R$ is not regular. (See also [20, Lemma 4.6.2].) Let $v \in \Delta$. Since $R$ is transitive on $\Delta$ we have $M_{0}^{\prime}=\left(M_{0}^{\prime}\right)_{v} R$ and it follows that $\left(M_{0}^{\prime}\right)_{v}=R_{v} .\left(M_{0}^{\prime} / R\right)$ with $1<R_{v}<R$. Also $R_{v} \cap Z=1$ and since $R$ acts irreducibly on $V_{0}$ we have $R_{v} Z \neq R$. Thus $\overline{R_{v}}=R_{v} Z / Z$ satisfies $1 \neq \overline{R_{v}}<\bar{R}$, and $\overline{R_{v}}$ is a normal subgroup of $\overline{\left(M_{0}^{\prime}\right)_{v}}$. Thus $\overline{R_{v}}$ is normalised by $\left\langle\overline{\left(M_{0}^{\prime}\right)_{v}}, \bar{R}\right\rangle=\overline{M_{0}^{\prime}}$, contradicting the fact that $\bar{R}$ is a minimal normal subgroup of $\overline{M_{0}^{\prime}}$. Thus $M_{0}^{\prime}$ acts non-trivially on the set of $R$-orbits.

As in [11, Chapter 5], for a finite group $G$, define

$$
P(G)=\min \{n \mid G \text { has a non-trivial permutation representation of degree } n\},
$$

and

$$
R_{r^{\prime}}(G)=\min \{n \mid G \leq \operatorname{PGL}(n, F), F \text { has characteristic coprime to } r\} .
$$

We prove the following extension of [11, Lemma 7.6.1]. (Note that we do not need this result to handle the case $d=3$.)

Lemma 4.6.7. Let $d \geq 4$ and when $d=4$ assume that $R$ is not of Type 2 . Then

$$
\begin{aligned}
& \text { 1. } P\left(\overline{M_{0}^{\prime}}\right) \geq d . \\
& \text { 2. } R_{r^{\prime}}\left(\overline{M_{0}^{\prime}}\right) \geq d \text {. }
\end{aligned}
$$

Proof. We follow the proof of [11, Lemma 7.6.1] but making various necessary adjustments, as [11, Lemma 7.6.1] applies only for $d \geq 13$. Let $X$ be a proper subgroup of $\overline{M_{0}^{\prime}}$. Suppose first that $\bar{R} X=\overline{M_{0}^{\prime}}$, so that $\bar{R} \not \leq X$ (since $X$ is a proper subgroup). Then, as $\bar{R} \cap X$ is normal in both $\bar{R}$ and $X$, it is normal in $\overline{M_{0}^{\prime}}$. Since $\bar{R}$ is a minimal normal subgroup of $\overline{M_{0}^{\prime}}$, and $\bar{R} \cap X \neq \bar{R}$, we have $\bar{R} \cap X=1$. Hence $\left|\overline{M_{0}^{\prime}}: X\right|=|\bar{R}|=r^{2 m}=d^{2}>d$, and part 1 holds in this case. Suppose now that $\bar{R} X \neq \overline{M_{0}^{\prime}}$. Then $\left|\overline{M_{0}^{\prime}}: X\right| \geq\left|\overline{M_{0}^{\prime}}: \bar{R} X\right| \geq P\left(M_{0}^{\prime} / R\right)$. As seen in the proof of [11, Lemma 7.6.1], when $d \geq 13$ we have $P\left(M_{0}^{\prime} / R\right)>d$, so that part 1 holds in these cases also. It remains to check the values $d=4,5,7,8,9$ and 11 . For $d=5,7$ or 11 , $P\left(M_{0}^{\prime} / R\right)=P(\operatorname{PSL}(2, d))=d$ (by [11, Table 5.2A], which we also use in the following). For $d=9, P\left(M_{0}^{\prime} / R\right)=P(\operatorname{PSp}(4,3))=27>d$. For $d=4$, note that $P\left(\operatorname{Sp}(4,2)^{\prime}\right)=6>d$ and $P\left(\Omega^{-}(4,2)\right)=5>d$. For $d=8$ we have $P(\operatorname{Sp}(6,2))=28, P\left(\Omega^{-}(6,2)\right)=27$ and $P\left(\Omega^{+}(6,2)\right)=8$. This completes the proof of part 1 .

Now we prove part 2. By [11, Lemma 5.5.3], either $R_{r^{\prime}}\left(\overline{M_{0}^{\prime}}\right) \geq \min \left\{P(\operatorname{Sp}(2 m, r)), r^{m}\right\}$ or $R_{r^{\prime}}\left(\overline{M_{0}^{\prime}}\right) \geq \min \left\{P\left(\Omega^{ \pm}(2 m, 2)\right), 2^{m}\right\}$. It follows from part 1 that $R_{r^{\prime}}\left(\overline{M_{0}^{\prime}}\right) \geq r^{m}=d$ and so the result follows. 
We now prove the following extension of [11, Proposition 7.6.2], the proof of which used the assumption $d \geq 13$, and had slightly more restrictive hypotheses on $M_{0}$.

Lemma 4.6.8. Suppose that $M_{0}<H \leq \mathrm{GL}\left(V_{0}\right)$. Then $\bar{H}:=H / Z_{p^{e}-1}$ is an almost simple group.

Proof. If $H$ leaves invariant, modulo scalars, a symplectic, unitary or quadratic form $\kappa$, let $X$ be the stabilizer in $\mathrm{GL}\left(V_{0}\right)$ of $\kappa$ modulo scalars, so $H \leq X$. In the case where $p=2$ and $H$ leaves invariant modulo scalars both a symplectic and a quadratic form, choose $\kappa$ to be the quadratic form. If $H$ leaves no such form invariant modulo scalars, then let $X=\mathrm{GL}\left(V_{0}\right)$. If $H$ contains the corresponding subgroup $X_{0}=\mathrm{Sp}\left(V_{0}\right), \operatorname{SU}\left(V_{0}\right), \Omega^{\varepsilon}\left(V_{0}\right)$ or $\mathrm{SL}\left(V_{0}\right)$, then $\bar{H}$ is almost simple. Thus we may assume that $H$ does not contain $X_{0}$. Because of this choice of classical group $X$, the subgroup $H$ is not in the class $\mathcal{C}_{8}$ for $X$. Moreover, since $R$ is absolutely irreducible and not writable over any proper subfield it follows that $H$ is not in the classes $\mathcal{C}_{1}, \mathcal{C}_{3}$ or $\mathcal{C}_{5}$ for $X$.

Suppose now that $H$ is in the class $\mathcal{C}_{2}$ for $X$. Then $M_{0}$ preserves a decomposition $V_{0}=U_{1} \oplus \ldots \oplus U_{t}$ for some $t \leq d$. Since $R$ is irreducible on $V_{0}$ it follows that $R$ is transitive on the decomposition. Moreover, $\bar{R}$ is a minimal normal subgroup of $\overline{M_{0}^{\prime}}$ and acts irreducibly on $V_{0}$ and hence acts faithfully on the set of $t$ parts of the decomposition. Since $|\bar{R}|=d^{2}$ and $\bar{R}$ is abelian this contradicts $t \leq d$. Thus $H \notin \mathcal{C}_{2}$.

Next suppose that $H$ lies in the class $\mathcal{C}_{4}$ or $\mathcal{C}_{7}$ for $X$. Then $M_{0}$ preserves a tensor decomposition $V_{0}=U_{1} \otimes \cdots \otimes U_{t}$ for some $t<d$ and each $U_{i}$ has dimension at least 2 . In particular $d$ is not prime so $d \geq 4$. By Lemma 4.6.7(1), $\overline{M_{0}^{\prime}} \leqslant \operatorname{PGL}\left(U_{1}\right) \times \cdots \times \operatorname{PGL}\left(U_{t}\right)$. Since $\bar{R}$ is a minimal normal subgroup of $\overline{M_{0}^{\prime}}$, it follows that $\bar{R}$ projects faithfully on at least one of the direct factors, so $\bar{R}$ is isomorphic to a subgroup of $\operatorname{PGL}\left(U_{i}\right)$, for some $i$. Moreover, since $\bar{R}$ is the unique minimal normal subgroup of $\overline{M_{0}^{\prime}}$, it follows that $\overline{M_{0}^{\prime}}$ also projects faithfully onto this factor, so $\overline{M_{0}^{\prime}}$ is isomorphic to a subgroup of $\operatorname{PGL}\left(U_{i}\right)$. This contradicts Lemma 4.6.7(2).

Finally, $H$ does not lie in the class $\mathcal{C}_{6}$ for $X$ by Lemma 4.6.5. It then follows from Aschbacher's Theorem [1] that $H$ lies in the class $\mathcal{C}_{9}$ for $X$, and in this case $\bar{H}$ is almost simple.

Now we complete the proof of Proposition 4.6.1.

Proof of Proposition 4.6.1. By Lemma 4.6.3, it is sufficient to prove the implication (4.6.5). We adopt the strategy of Remark 4.6.4. Thus we suppose that $M_{0}<H \leq \mathrm{GL}\left(V_{0}\right)$ and that $H$ permutes amongst themselves the $R$-orbits in $V_{0}$. Note in particular that $\mathrm{SL}\left(V_{0}\right) H$ since $\mathrm{SL}\left(V_{0}\right)$ is 2-transitive on the 1-spaces in $V_{0}$ and hence does not permute the $R$-orbits among themselves. It is sufficient to derive a contradiction for each choice of the group $R \in \mathcal{R}$. By Subsection 4.6.2, we may assume that $d \geq 3$, and if $d=4$ then $R$ is not of Type 2 . In each of these cases, $M_{0}^{\prime}$ acts non-trivially on the set of $R$-orbits in $V_{0}$ by Lemma 4.6.6. Also either $\overline{M_{0}^{\prime}}$ is perfect or $d=3, \overline{M_{0}}=Z_{3}^{2}: \operatorname{Sp}(2,3)$, and $\overline{M_{0}^{\prime}}=Z_{3}^{2}: Q_{8}$. Moreover, by Lemma 4.6.8, $\bar{H}=H / Z_{p^{e}-1}$ is an almost simple group with nonabelian simple socle $\bar{T}$, say, so that $\bar{H} / \bar{T}$ is soluble and $\operatorname{PSL}\left(d, p^{e}\right) \neq \bar{T}$. 
Let $Z_{p^{e-1}}<T \leq H$ be such that $T / Z_{p^{e}-1}=\bar{T}$. Suppose first of all that $d=3$, and note that $e=1$ or $2, \overline{M_{0}^{\prime}}=Z_{3}^{2}: Q_{8} \leqslant \operatorname{PSL}\left(3, p^{e}\right)$, and $3=\left|\overline{M_{0}}: \overline{M_{0}^{\prime}}\right|$ so that $3^{3}$ divides $|\bar{H}|$. If $q$ is even then $p^{e}=4$ and we see from $[3, \mathrm{p} 23]$ that $\overline{M_{0}}$ is maximal in PGL $(3,4)$ so that $\operatorname{SL}(3,4) \leqslant H$ which is a contradiction. Hence $q$ is odd. The subgroups of PSL $(3, q)$ for $q$ odd were determined by Mitchell and are given in [8, Theorem 2.4]. Since $e$ is minimal such that $p^{e} \equiv 1(\bmod \mid) Z(R) \mid$, the only possibilities for $\bar{T}$ are $\operatorname{PSU}(3, p)$ (when $e=2$ ) and $A_{6}$. Since $3^{3}$ does not divide $\left|\operatorname{Aut}\left(A_{6}\right)\right|$ we cannot have the latter and so $\bar{T}=\operatorname{PSU}(3, p)$. Moreover, in this case $\bar{T}$ contains $\overline{M_{0}^{\prime}}$, see $[8$, Theorem 2.6]. Hence $T$ contains $M_{0}^{\prime}$ and so by Lemma 4.6.6, $T$ acts non-trivially on the set of $R$-orbits. Since $\bar{T}$ is simple, it follows that the kernel of this action is contained in the scalars $Z_{p^{e}-1}$. This is a contradiction since $R$ fixes each of its orbits setwise and yet does not consist of scalars. Thus $d \neq 3$, and in particular $\overline{M_{0}^{\prime}}$ is perfect. Since $\bar{H} / \bar{T}$ is soluble, it follows that $\bar{T}$ contains $\overline{M_{0}^{\prime}}$. Thus $T$ contains $M_{0}^{\prime}$, and by Lemma 4.6.6, $T$ acts non-trivially on the set of $R$-orbits in $V_{0}$. Since $\bar{T}$ is simple, it follows that the kernel of this action is contained in the scalars $Z_{p^{e}-1}$. Once again this is a contradiction since $R$ fixes each of its orbits setwise. This completes the proof.

\subsection{The Case $H \in \mathcal{C}_{8}$}

As described in Subsection 2.2, the family of $\mathcal{C}_{8}$-subgroups is the union of three subfamilies $\mathcal{C}_{\mathbf{X}}$, for $\mathbf{X} \in\{\mathbf{S p}, \mathbf{U}, \mathbf{O}\}$. The sub-family $\mathcal{C}_{\mathbf{X}}$ consists of all subgroups that preserve modulo scalars an $\mathbf{X}$-form on $V$ defined as follows.

A form $\mathbf{f}$ is an $\mathbf{S p}$-form (or symplectic form) if it is bilinear $\mathbf{f}: V \times V \rightarrow F_{q}$, nondegenerate and skew symmetric, and if in addition $q$ is even then $\mathbf{f}(v, v)=0$ for all $v \in V$. For such a form $d$ is even and we define $\Delta_{\mathbf{f}}:=\{(u, v) \mid \mathbf{f}(u, v)=0\}$,

$$
k_{\mathbf{S p}}=2 \quad \text { and } \operatorname{Rel}(\mathbf{S p}, 2)=\left\{\begin{array}{cl}
\left\{\Delta_{\mathbf{f}} \mid \mathbf{f} \text { a symplectic form on } V\right\} & \text { if } d \text { is even } \\
\emptyset & \text { if } d \text { is odd }
\end{array}\right.
$$

A form $\mathbf{Q}$ is an $\mathbf{O}$-form if it is a quadratic form $\mathbf{Q}: V \rightarrow F_{q}$ (that is, $\mathbf{Q}(\lambda v)=\lambda^{2} \mathbf{Q}(v)$ for all $v \in V$ and $\lambda \in F_{q}$ ) and is non-degenerate, that is, the associated bilinear form

$$
\mathbf{f}_{\mathbf{Q}}(v, w):=\mathbf{Q}(v+w)-\mathbf{Q}(v)-\mathbf{Q}(w)
$$

is non-degenerate. If $d$ is even, say $d=2 m$, there are two types of forms with different stabilizers in $\Gamma \mathrm{L}(d, q)$, namely +-type forms for which maximal totally singular subspaces have dimension $m$, and --type forms for which such subspaces have dimension $m-1$. In particular if $d=2$ then the --type forms $\mathbf{Q}$ have no $\mathbf{Q}$-singular vectors (non-zero vectors $v$ such that $\mathbf{Q}(v)=0$ ); as mentioned in Remark 2.2.1(a), the stabilizers of such forms modulo scalars are maximal $\mathcal{C}_{3}$-subgroups and are treated as such. We assume that $\mathbf{Q}$ has +-type if $d=2$. If $d$ is odd there is only one $\Gamma \mathrm{L}(d, q)$-conjugacy class of stabilizers of O-forms. For an $\mathbf{O}$-form $\mathbf{Q}$ we define $\Delta_{\mathbf{Q}}:=\{v \mid \mathbf{Q}(v)=0\}$,

$$
k_{\mathbf{O}}=1 \text { and } \operatorname{Rel}(\mathbf{O}, 1)=\left\{\Delta_{\mathbf{Q}} \mid \mathbf{f} \text { an } \mathbf{O} \text {-form on } V\right\} .
$$


A form $\mathbf{f}$ is a $\mathbf{U}$-form (or unitary form) if the field order $q=q_{0}^{2}$ so that $\alpha: \lambda \rightarrow \lambda^{q_{0}}$ is an involutory automorphism of $F_{q}$, and if $\mathbf{f}: V \times V \rightarrow F_{q}$ is non-degenerate and hermetian symmetric (that is, $\mathbf{f}$ is left-linear and $\mathbf{f}(w, v)=\mathbf{f}(v, w)^{\alpha}$ for all $v, w \in V$ ). For such a form we define $\Delta_{\mathbf{f}}:=\{v \mid \mathbf{f}(v, v)=0\}$,

$$
k_{\mathbf{U}}=1 \quad \text { and } \operatorname{Rel}(\mathbf{U}, 1)=\left\{\begin{array}{cl}
\left\{\Delta_{\mathbf{f}} \mid \mathbf{f} \text { a unitary form on } V\right\} & \text { if } q \text { is a square } \\
\emptyset & \text { if not. }
\end{array}\right.
$$

Proposition 4.7.1. For $\mathbf{X} \in\{\mathbf{S p}, \mathbf{U}, \mathbf{O}\}, H \in \mathcal{C}_{\mathbf{X}}$ if and only if $\operatorname{Rel}\left(H, k_{\mathbf{X}}\right) \cap \operatorname{Rel}\left(\mathbf{X}, k_{\mathbf{X}}\right)$ $\neq \emptyset$.

More precisely, when we say that an element $g \in \Gamma \mathrm{L}(d, q)$ 'preserves an $\mathbf{X}$-form $\mathbf{f}$ modulo scalars' we mean that $g$ is an f-semisimilarity, that is, there exist $\lambda \in F_{q}^{*}$ and $\sigma \in \operatorname{Aut}\left(F_{q}\right)$ such that $\mathbf{f}\left(u^{g}, v^{g}\right)=\lambda \mathbf{f}(u, v)^{\sigma}$ for all $u, v \in V$ in case $\mathbf{S p}$ or $\mathbf{U}$, or $\mathbf{f}\left(v^{g}\right)=$

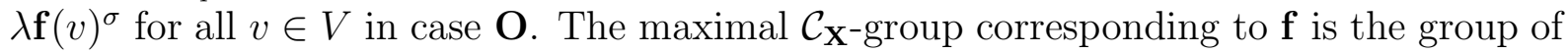
all f-semisimilarities.

Regarding the proof of Proposition 4.7.1, it follows from the definition of the relation sets that, if $H \in \mathcal{C}_{\mathbf{X}}$ then $H$ consists of $\mathbf{f}$-semisimilarities for some $\mathbf{X}$-form $\mathbf{f}$, and hence that $H$ leaves $\Delta_{\mathbf{f}}$ invariant. Thus to prove Proposition 4.7.1, we assume conversely that $H$ preserves a relation $\Delta_{\mathbf{f}} \in \operatorname{Rel}\left(\mathbf{X}, k_{\mathbf{X}}\right)$, for some $\mathbf{X}$, and we prove that $H$ consists of f-semisimilarities. We do this separately for each $\mathbf{X} \in\{\mathbf{S p}, \mathbf{U}, \mathbf{O}\}$.

\subsubsection{The symplectic groups}

Since $\Delta_{\mathbf{f}} \in \operatorname{Rel}(\mathbf{S p}, 2), d=2 m$, and $V$ has a 'symplectic basis' $\left\{e_{1}, \ldots, e_{m}, f_{1}, \ldots, f_{m}\right\}$ such that for all $i, j, \mathbf{f}\left(e_{i}, e_{j}\right)=\mathbf{f}\left(f_{i}, f_{j}\right)=0$ and $\mathbf{f}\left(e_{i}, f_{j}\right)=\delta_{i j}$. Then $\Delta_{\mathbf{f}}$ contains $\left(e_{i}, e_{j}\right)$ and $\left(f_{i}, f_{j}\right)$ for all $i, j$, and $\left(e_{i}, f_{j}\right)$ for $i \neq j$. Let $g \in H$. Then since $g$ preserves $\Delta_{\mathbf{f}}$, the relation $\Delta_{\mathbf{f}}$ also contains the images of all these pairs under $g$, and hence

$$
\mathbf{f}\left(e_{i}^{g}, e_{j}^{g}\right)=\mathbf{f}\left(f_{i}^{g}, f_{j}^{g}\right)=0 \text { for all } i, j \text {, and } \mathbf{f}\left(e_{i}^{g}, f_{j}^{g}\right)=0 \text { when } i \neq j \text {. }
$$

Since $\mathbf{f}\left(e_{1}, f_{1}\right)=1$, the pair $\left(e_{1}, f_{1}\right) \notin \Delta_{\mathbf{f}}$ and hence $\left(e_{1}^{g}, f_{1}^{g}\right) \notin \Delta_{\mathbf{f}}$. Thus $\lambda:=\mathbf{f}\left(e_{1}^{g}, f_{1}^{g}\right) \neq 0$. Since, for $i>1, \mathbf{f}\left(-e_{1}+e_{i}, f_{1}+f_{i}\right)=-1+1=0$, we have

$$
0=\mathbf{f}\left(-e_{1}^{g}+e_{i}^{g}, f_{1}^{g}+f_{i}^{g}\right)=-\mathbf{f}\left(e_{1}^{g}, f_{1}^{g}\right)+\mathbf{f}\left(e_{i}^{g}, f_{i}^{g}\right)=-\lambda+\mathbf{f}\left(e_{i}^{g}, f_{i}^{g}\right) .
$$

Therefore $\mathbf{f}\left(e_{i}^{g}, f_{i}^{g}\right)=\lambda$ for all $i$. For arbitrary $u=\sum_{i=1}^{m}\left(\mu_{i} e_{i}+\mu_{i}^{\prime} f_{i}\right)$ and $v=\sum_{i=1}^{m}\left(\nu_{i} e_{i}+\right.$ $\left.\nu_{i}^{\prime} f_{i}\right)$ in $V$, we have, since $\mathbf{f}\left(f_{j}, e_{i}\right)=-\mathbf{f}\left(e_{i}, f_{j}\right)=-\delta_{i j}$, that

$$
\mathbf{f}(u, v)=\sum_{i=1}^{m}\left(\mu_{i} \nu_{i}^{\prime} \mathbf{f}\left(e_{i}, f_{i}\right)+\mu_{i}^{\prime} \nu_{i} \mathbf{f}\left(f_{i}, e_{i}\right)\right)=\sum_{i=1}^{m}\left(\mu_{i} \nu_{i}^{\prime}-\mu_{i}^{\prime} \nu_{i}\right) .
$$

Let $\sigma=\tau(g)$ (as defined in Subsection 2.1). Then $u^{g}=\sum_{i=1}^{m}\left(\mu_{i}^{\sigma} e_{i}^{g}+\mu_{i}^{\sigma} f_{i}^{g}\right), v^{g}=$ $\sum_{i=1}^{m}\left(\nu_{i}^{\sigma} e_{i}^{g}+\nu_{i}^{\prime \sigma} f_{i}^{g}\right)$, and

$$
\mathbf{f}\left(u^{g}, v^{g}\right)=\sum_{i=1}^{m}\left(\left(\mu_{i} \nu_{i}^{\prime}\right)^{\sigma} \mathbf{f}\left(e_{i}^{g}, f_{i}^{g}\right)+\left(\mu_{i}^{\prime} \nu_{i}\right)^{\sigma} \mathbf{f}\left(f_{i}^{g}, e_{i}^{g}\right)\right)=\lambda \sum_{i=1}^{m}\left(\mu_{i} \nu_{i}^{\prime}-\mu_{i}^{\prime} \nu_{i}\right)^{\sigma}=\lambda \mathbf{f}(u, v)^{\sigma} .
$$


Therefore $g$ is an f-semisimilarity and hence $H$ is contained in the maximal $\mathcal{C}_{\mathbf{S p}}$-group consisting of $\mathbf{f}$-semisimilarities.

\subsubsection{The orthogonal groups}

For the orthogonal case we write $\mathbf{Q}$ instead of $\mathbf{f}$, and speak also of the corresponding bilinear form $\mathbf{f}_{\mathbf{Q}}$ defined in (4.7.2). We assume that $H$ preserves the (non-empty) relation $\Delta_{\mathbf{Q}} \in \operatorname{Rel}(\mathbf{O}, 1)$ (recall if $d=2$ then $\mathbf{Q}$ is of +-type.) Let $g \in H$. We will prove that $g$ is a Q-semisimilarity. Our proof is a modification of [10, Lemma 1] suggested to us by Dr. Oliver King, and we are grateful to him for this. The result [10, Lemma 1] proves what we need in the special case where $H \subseteq \mathrm{GL}(d, q)$ and $q$ is odd.

We subdivide the set of 2-subspaces $U$ of $V$ that contain a $\mathbf{Q}$-singular vector. We write $U^{\perp}$ for the orthogonal complement $\left\{w \in V \mid \mathbf{f}_{\mathbf{Q}}(u, w)=0\right.$ for all $\left.u \in U\right\}$. If $\operatorname{dim}\left(U^{\perp} \cap U\right)=$ 0 or 1 , then $U$ is in case 1 or 2 below, respectively, while if $\operatorname{dim}\left(U^{\perp} \cap U\right)=2$ then $U$ may or may not be totally singular, and satisfies case 3 or 2 below, respectively.

1. Non-degenerate: $U \cap U^{\perp}=0$; here $U$ has +-type, $\left|U \cap \Delta_{\mathbf{Q}}\right|=2(q-1)+1$, and $v^{\perp} \cap U=\langle v\rangle$ for $v \in\left(U \cap \Delta_{Q}\right) \backslash\{0\}$.

2. Tangent: $U \cap \Delta_{\mathbf{Q}}=\langle v\rangle$ so $\left|U \cap \Delta_{\mathbf{Q}}\right|=q$; and $U \subseteq v^{\perp}$. In fact either $U^{\perp} \cap U=U \cap \Delta_{\mathbf{Q}}$, or $q$ is even and $U \subseteq U^{\perp}$.

3. Totally singular: $U \subseteq \Delta_{\mathbf{Q}}$ so $\left|U \cap \Delta_{\mathbf{Q}}\right|=q^{2}$. Here $U \subseteq v^{\perp}$ for $0 \neq v \in U$.

Since the sizes $\left|U \cap \Delta_{\mathbf{Q}}\right|$ are pairwise distinct for the three cases, and since $g$ preserves $\Delta_{\mathbf{Q}}$, it follows that $g$ preserves the above three kinds of 2 -subspaces. We record a few easy facts about the $g$-action.

Lemma 4.7.2. Suppose that $U=\langle u, v\rangle$ is a 2-subspace and $\mathbf{Q}(v)=0$.

(a) If $\mathbf{f}_{\mathbf{Q}}(u, v)=0$ then $U$ is not non-degenerate.

(b) If $U$ is not non-degenerate, then $U \subseteq v^{\perp}$ and $U^{g} \subseteq\left(v^{g}\right)^{\perp}$.

Proof. If $\mathbf{f}_{\mathbf{Q}}(u, v)=0$ then $U \subseteq v^{\perp}$, so $U$ is not non-degenerate. Next suppose that $U$ is either a tangent or totally singular kind of 2-space. Then $U \subseteq v^{\perp}$. Since $g$ preserves $\Delta_{\mathbf{Q}}$ and preserves these three kinds of 2-spaces, $v^{g} \in U^{g}$ is a $\mathbf{Q}$-singular vector and $U^{g}$ is tangent or totally singular, so $U^{g} \subseteq\left(v^{g}\right)^{\perp}$.

Since $\Delta_{\mathbf{Q}}$ is non-empty, $V$ has a non-degenerate 2-subspace $U$ of +-type, so $U=\langle e, f\rangle$ where $\mathbf{Q}(e)=\mathbf{Q}(f)=0$ and $\mathbf{f}_{\mathbf{Q}}(e, f)=\mathbf{Q}(e+f)=1$ (see [18, 11.3]). Set $e_{1}:=e^{g}$ and $f_{1}:=f^{g}$. Since $g$ preserves $\Delta_{\mathbf{Q}}, \mathbf{Q}\left(e_{1}\right)=\mathbf{Q}\left(f_{1}\right)=0$, and as $g$ preserves non-degenerate 2 -spaces of +-type, $U^{g}$ is non-degenerate of +-type. Hence $\mathbf{f}_{\mathbf{Q}}\left(e_{1}, f_{1}\right)=\lambda$ for some $\lambda \in F_{q}^{*}$. For an arbitrary $v=\mu e+\nu f \in U$, we have $\mathbf{Q}(v)=\mu \nu$ and, writing $\sigma=\tau(g)$ for the associated field automorphism of $g$,

$$
\mathbf{Q}\left(v^{g}\right)=\mathbf{Q}\left(\mu^{\sigma} e_{1}+\nu^{\sigma} f_{1}\right)=\mathbf{f}_{\mathbf{Q}}\left(\mu^{\sigma} e_{1}, \nu^{\sigma} f_{1}\right)=\lambda(\mu \nu)^{\sigma}=\lambda \mathbf{Q}(v)^{\sigma} .
$$


For $w \in U^{\perp}$, by Lemma 4.7.2 (a), the 2-subspaces $\langle e, w\rangle$ and $\langle f, w\rangle$ are not non-degenerate, and so by Lemma 4.7.2 (b), $\left\langle e_{1}, w^{g}\right\rangle \subseteq\left\langle e_{1}\right\rangle^{\perp}$ and $\left\langle f_{1}, w^{g}\right\rangle \subseteq\left\langle f_{1}\right\rangle^{\perp}$. Hence $w^{g} \in\left\langle e_{1}, f_{1}\right\rangle^{\perp}$, and as this holds for all $w \in U^{\perp}$, we have $\left(U^{\perp}\right)^{g} \subseteq\left(U^{g}\right)^{\perp}$. It follows that $\left(U^{\perp}\right)^{g}=\left(U^{g}\right)^{\perp}$.

Consider the vector $x=w+e-\mathbf{Q}(w) f$ where $w \in U^{\perp}$. Using the properties of $\mathbf{Q}, \mathbf{Q}(x)=\mathbf{Q}(w)+\mathbf{Q}(e-\mathbf{Q}(w) f)=0$, and therefore, since $g$ preserves $\Delta_{\mathbf{Q}}$ and since $w^{g} \in\left(U^{g}\right)^{\perp}$

$$
0=\mathbf{Q}\left(x^{g}\right)=\mathbf{Q}\left(w^{g}\right)+\mathbf{Q}\left(e_{1}-\mathbf{Q}(w)^{\sigma} f_{1}\right)=\mathbf{Q}\left(w^{g}\right)-\lambda \mathbf{Q}(w)^{\sigma} .
$$

Thus $\mathbf{Q}\left(w^{g}\right)=\lambda \mathbf{Q}(w)^{\sigma}$, and this holds for any $w \in U^{\perp}$. A typical vector of $V$ is of the form $v+w$ with $v \in U$ and $w \in U^{\perp}$. Now $\mathbf{Q}(v+w)=\mathbf{Q}(v)+\mathbf{Q}(w)$, and since $\left(U^{\perp}\right)^{g}=\left(U^{g}\right)^{\perp}$, we have $\mathbf{f}_{\mathbf{Q}}\left(v^{g}, w^{g}\right)=0$, and hence

$$
\mathbf{Q}\left((v+w)^{g}\right)=\mathbf{Q}\left(v^{g}\right)+\mathbf{Q}\left(w^{g}\right)=\lambda \mathbf{Q}(v)^{\sigma}+\lambda \mathbf{Q}(w)^{\sigma}=\lambda \mathbf{Q}(v+w)^{\sigma} .
$$

Therefore $g$ is a $\mathbf{Q}$-semisimilarity. Thus we conclude that $H$ is contained in the maximal

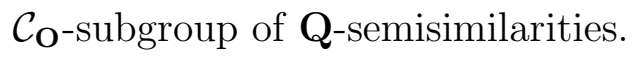

\subsubsection{The unitary groups}

We assume here that $H$ leaves $\Delta_{\mathbf{f}}$ invariant for some unitary form $\mathbf{f}$. Let $g \in H$. We must prove that $g$ is an $\mathbf{f}$-semisimilarity. Now the subgroup $X$ of all $\mathbf{f}$-semisimilarities in $\Gamma \mathrm{L}(d, q)$ satisfies $\Gamma \mathrm{L}(d, q)=X(\mathrm{GL}(d, q))$, and hence there is an f-semisimilarity $h$ such that $\tau(g)=\tau(h)$ (the field automorphism induced by these elements). Thus $g h^{-1} \in$ $\operatorname{GL}(d, q)$ and $g h^{-1}$ leaves $\Delta_{\mathbf{f}}$ invariant. It follows from [9, Proposition 1] that $g h^{-1}$ is an $\mathbf{f}$-similarity, and hence $g$ is an $\mathbf{f}$-semisimilarity. Thus $H$ is contained in the maximal $\mathcal{C}_{\mathbf{U}}$-subgroup of $\mathbf{f}$-semisimilarities.

This completes the proof of Proposition 4.7.1.

Proof of Theorem 1.1. Theorem 1.1 now follows from Propositions 4.1.1, 4.2.1, 4.3.1, 4.4.1, 4.5.1, 4.6.1, and 4.7.1.

\section{Proof of Theorem 1.4}

In this section let $G$ be an affine primitive permutation group on a finite set $\Omega$, so $G=N H$ with $N=Z_{p}^{d}$ the group of translations of a finite vector space $V=F_{p}^{d}$ and $H \leq \mathrm{GL}(d, p)$, where $d \geq 1$ and $p$ is a prime. We identify $\Omega$ with $V$. If one of $d \leq 2$, or $\operatorname{SL}(d, p) \leq H$, or $H \in \mathcal{C}_{9}$, then the assertions made about such groups in Theorem 1.4 all hold, by Lemma 3.3.2. Thus we may assume that $d \geq 3$, that $H$ does not contain $\operatorname{SL}(d, p)$, and that $H \in \mathcal{C}_{i}$ for some $i \in\{1, \ldots, 7, \mathbf{S p}, \mathbf{U}, \mathbf{O}\}$.

Suppose first that $G^{(3)}$ is an affine primitive group. Then $G^{(3)}=N K$ where $H \leq$ $K \leq \mathrm{GL}(d, p)$. By Lemma 2.3.1(4), $H \leq K \leq H^{(2)}$, so $K \leq H^{(2)} \cap \mathrm{GL}(d, p)$. By Corollary 1.2, $H^{\left(k_{i}\right)} \cap \mathrm{GL}(d, p) \in \mathcal{C}_{i}$ also. Since $H^{(2)} \leq H^{(1)}$, by Lemma 2.3.1(1), this 
implies that, in all cases, $H^{(2)} \cap \mathrm{GL}(d, p) \in \mathcal{C}_{i}$. Hence $K \in \mathcal{C}_{i}$, and then by Theorem 1.1, $\operatorname{Rel}\left(K, k_{i}\right) \cap \operatorname{Rel}\left(i, k_{i}\right) \neq \emptyset$. Thus Theorem 1.4(b)(iii) holds.

Thus we may assume that $G^{(3)}$ is not an affine primitive group. We denote the socle of a finite group $X$ by $\operatorname{Soc}(X)$ (the product of its minimal normal subgroups). Thus, $\operatorname{Soc}(G)=N \neq \operatorname{Soc}\left(G^{(3)}\right)$. Let $G<L \leq G^{(3)}$ be such that the socle $\operatorname{Soc}(L) \neq N$. Then the following result [14, Lemma 4.1] of Saxl and the fourth author applies. The result refers to primitive permutation groups in product action, as discussed in Subsection 2.5.

lemma 5.1. [14, Lemma 4.1] Let $G=N H, d, L, G^{(3)}$ be as above. Then $p=2$ and one of the following holds.

(a) $(d, H, \operatorname{Soc}(L))=\left(4, A_{7}, A_{16}\right)$ as in Theorem 1.4(a), Line 2 of Table 2.

(b) $d \geq 3$ and $(H, \operatorname{Soc}(L))=\left(\mathrm{GL}(d, 2), A_{2^{d}}\right)$ as in Theorem 1.4(a), Line 1 of Table 2 .

(c) L preserves a product decomposition $\Gamma^{m}$ of $\Omega$, where $|\Gamma|=2^{n}, d=n m, m \geq 2$, and the permutation group $G_{0}$ induced by $G$ on $\Gamma$ is $Z_{2}^{4} \rtimes A_{7}$ (with $\left.n=4\right)$ or $\operatorname{AGL}(n, 2)$ (with $n \geq 3$ ). Moreover, the group induced by $L$ on $\Gamma$ contains $A_{2^{n}}$ and

$$
\operatorname{Soc}(L)=\operatorname{Soc}\left(G^{(3)}\right) \cong \overbrace{A_{2^{n}} \times \ldots \times A_{2^{n}}}^{m} .
$$

To complete the proof we may therefore assume that Lemma 5.1(c) holds. Thus $L \leq L_{0} 2 S_{m}$ acting in product action on $\Omega=\Gamma^{m}$, $\operatorname{Soc}(L)=A_{2^{n}}^{m}$, and the permutation group $G_{0}$ induced by $G$ on $\Gamma$ is either $Z_{2}^{4} \rtimes A_{7}$ (with $\left.n=4\right)$ or $\operatorname{AGL}(n, 2)$ (with $n \geq 3$ ). It follows, from the discussion of product action in Subsection 2.5, that we may take $G \leq G_{0} \zeta Y$, where $Y=\pi(G)$, the projection of $G$ on $S_{m}$, is a transitive subgroup of $S_{m}$. We will prove that $G$ contains the base group $G_{0}^{m}$ of the wreath product $G_{0} 2 S_{m}$. This will imply that $G=G_{0} \backslash Y$, and that $L$ contains $A_{2^{n}} \backslash Y$, so that Theorem 1.4(a) holds (Line 3 or 4 of Table 2), thus completing the proof.

Consider the stabilizer $G_{\alpha}$ of the point $\alpha=(\delta, \ldots, \delta) \in \Gamma^{m}$. We have $G_{\alpha} \leq\left(G_{0}\right)_{\delta} 2 S_{m}$, and the point stabilizer $\left(G_{0}\right)_{\delta} \cong \mathrm{GL}(n, 2)$ (with $2^{n} \geq 8$ ) or $A_{7}$ (with $n=4$ ). In particular, $\left(G_{0}\right)_{\delta}$ is a nonabelian simple group.

lemma 5.2. $\left(2^{n}-1\right)^{m}\left(2^{n}-2\right)^{m}$ divides $\left|G_{\alpha}\right|$ where $\alpha=(\delta, \ldots, \delta) \in \Gamma^{m}$.

Proof. First, consider the action of $G^{(3)}$. Since $\operatorname{Soc}\left(G^{(3)}\right)=A_{2^{n}}^{m} \leq G^{(3)}$, the point stabilizer $\left(G^{(3)}\right)_{\alpha} \geq\left(A_{2^{n}-1}\right)^{m}$ where $2^{n}-1 \geq 7$. Let $\mu, \nu$ be distinct points in $\Gamma \backslash\{\delta\}$, let $\beta=$ $(\mu, \ldots, \mu) \in \Gamma^{m}$ and $\gamma=(\nu, \ldots, \nu) \in \Gamma^{m}$. Let $\Delta$ be the orbit of $\left(A_{2^{n}-1}\right)^{m}$ containing the pair $(\beta, \gamma)=((\mu, \ldots, \mu),(\nu, \ldots, \nu))$. Since $A_{2^{n}-1}$ is 2 -transitive on $\Gamma \backslash\{\delta\}$, we have

$$
\Delta=\left\{\left(\left(\mu_{1}, \ldots, \mu_{m}\right),\left(\nu_{1}, \ldots, \nu_{m}\right)\right) \mid \mu_{i}, \nu_{i} \in \Gamma \backslash\{\delta\} \text { and } \mu_{i} \neq \nu_{i} \text { for all } i \leq m\right\} .
$$

Since this subset is invariant under $\left(G^{(3)}\right)_{\alpha}$, it follows that $\Delta$ is a $\left(G^{(3)}\right)_{\alpha}$-orbit. By Lemma 2.3.1 (4), $G_{\alpha}$ is 2-equivalent to $\left(G^{(3)}\right)_{\alpha}$, and so $\Delta$ is also a $G_{\alpha}$-orbit. Hence $\left|G_{\alpha}\right|$ is divisible by $|\Delta|=\left(2^{n}-1\right)^{m}\left(2^{n}-2\right)^{m}$. 
A prime $s$ dividing $2^{n}-1$ is said to be a primitive prime divisor of $2^{n}-1$ if $s$ does not divide $2^{i}-1$ for any $i$ such that $1 \leq i<n$. By Zsigmondy [21], and since $n \geq 3,2^{n}-1$ has a primitive prime divisor unless $n=6$.

Let $s$ be a primitive prime divisor of $2^{n}-1$ if $n \neq 6$, and let $s=31=2^{5}-1$ if $n=6$. Let $s^{a}$ be the highest power of $s$ dividing $\left(2^{n}-1\right)\left(2^{n-1}-1\right)$. Then $a \geq 1$ and, by Lemma $5.2, s^{a m}$ divides $\left|G_{\alpha}\right|$. Let

$$
M=G_{\alpha} \cap \overbrace{\left(\left(G_{0}\right)_{\delta} \times \ldots \times\left(G_{0}\right)_{\delta}\right)}^{m} .
$$

Then $M$ is the intersection of $G_{\alpha}$ with the base group of $G_{0} \prec S_{m}$, and hence $M \unlhd G_{\alpha}$ and $G_{\alpha} / M \cong Y \leq S_{m}$. Now the highest power of $s$ dividing $\left|S_{m}\right|=m$ ! is $s^{c}$ where $c \leq\left[\frac{m-1}{s-1}\right] \leq\left[\frac{m-1}{2}\right]$ (see, for example, [5, Exercise 2.6.8]). Then since $s^{a m}$ divides $\left|G_{\alpha}\right|$, it follows that $s^{a m-\left[\frac{m-1}{2}\right]}$ divides $|M|$. In particular, since $a m-\left[\frac{m-1}{2}\right] \geq 1$, the group $M$ is non-trivial.

Let $T:=\left(G_{0}\right)_{\delta}$, and recall that $T=\mathrm{GL}(n, 2)$ or $A_{7}$, a nonabelian simple group, and write

$$
\overbrace{\left(G_{0}\right)_{\delta} \times \ldots \times\left(G_{0}\right)_{\delta}}^{m}=T_{1} \times \ldots \times T_{m} \text { where } T_{i}=T=\left(G_{0}\right)_{\delta} .
$$

Next we prove that $M \cong T^{u}$, for some $u$ dividing $m$.

Recall from Subsection 2.5 the subgroups

$$
W_{i}=\operatorname{Sym}\left(\Gamma_{i}\right) \times\left(\operatorname{Sym}(\Gamma) \imath S_{m-1}\right)
$$

and projection maps $\pi_{i}: G \cap W_{i} \rightarrow \operatorname{Sym}\left(\Gamma_{i}\right)$ and note that $M \leq W_{i}$ for all $i$. Since $G \leq G_{0} 2 S_{m}$, for each $i$,

$$
\pi_{i}\left(G_{\alpha} \cap W_{i}\right)=\left(\pi_{i}\left(G \cap W_{i}\right)\right)_{\delta} \cong\left(G_{0}\right)_{\delta} .
$$

Since $Y=\pi(G)=\pi\left(G_{\alpha}\right) \leq S_{m}$ is transitive, it follows that $G_{\alpha}$ acts transitively on $\Sigma=\left\{T_{1}, \ldots, T_{m}\right\}$ by conjugation. Let $i, j \in\{1, \ldots, m\}$. Then there exists $x \in G_{\alpha}$ such that $\pi(x): i \rightarrow j$. Since $M \triangleleft G_{\alpha}, x^{-1} \pi_{i}(M) x=\pi_{j}(M)$. Thus $\pi_{i}(M) \cong \pi_{j}(M)$ for $1 \leq i, j \leq m$. Now, the facts that $\{1\} \neq M \unlhd\left(G_{\alpha} \cap W_{i}\right)$ and $\left(G_{0}\right)_{\delta}=T$ is simple together with (5.0.5). imply that

$$
\pi_{i}(M) \cong\left(G_{0}\right)_{\delta}=T \quad \text { for } i=1,2, \ldots, m .
$$

By [5, Lemma 4.3A], there exists a partition $\left\{\Lambda_{1}, \ldots, \Lambda_{u}\right\}$ of $\{1, \ldots, m\}$ such that $M=$ $D_{1} \times \ldots \times D_{u}$ where $D_{i} \cong T$ is a full diagonal subgroup of the subproduct $\prod_{j \in \Lambda_{i}} T_{j}$. Since $M \triangleleft G_{\alpha}$ and $G_{\alpha}$ acts on $\Sigma$ transitively, the integer $u$ divides $m$ and each $\left|\Lambda_{i}\right|=m / u$.

Finally we prove that $u=m$. Now

$$
|\mathrm{GL}(n, 2)|=2^{\frac{n(n-1)}{2}} \prod_{i=1}^{n}\left(2^{i}-1\right) .
$$

By the choice of $s, s^{a u}$ is the highest power of $s$ that divides $\left|\operatorname{GL}(n, 2)^{u}\right|$. Therefore the power of $s$ that divides $|M|=|T|^{u}$ is at most $s^{a u}$. On the other hand, we showed above 
that $s^{a m-\left[\frac{m-1}{2}\right]}$ divides $|M|$, and hence $a u \geq a m-\left[\frac{m-1}{2}\right]>\left(a-\frac{1}{2}\right) m$. This implies that $u>\frac{m}{2}$, and since $u$ divides $m$, we conclude that $u=m$. Thus $M=G_{0}^{m} \leq G$, and hence, as discussed above, $G=G_{0} \imath Y$, completing the proof of Theorem 1.4.

\section{References}

[1] M. Aschbacher, On the maximal subgroups of the finite classical groups, Invent. Math. 76 (1984), 469-514.

[2] P. J. Cameron and W. M. Kantor, 2-transitive and antiflag transitive collineation groups of finite projective spaces, J. Algebra 60 (1979), 384-422.

[3] J. H. Conway, R. T. Curtis, S. P. Norton, R. A. Parker and R. A. Wilson, Atlas of finite groups, Clarendon Press, Oxford, 1985.

[4] L. E. Dickson, Linear Groups with an Exposition of the Galois Field Theory, Leibzig: Teubner 1901 (New York: Dover Publ. 1958).

[5] J. D. Dixon and B. Mortimer, Permutation Groups, Springer-Verlag, New YorkHeidelberg-Berlin, 1996.

[6] G. Hiss and G. Malle, Corrigenda: "Low-dimensional representations of quasi-simple groups" [LMS J. Comput. Math. 4 (2001), 22-63]. LMS J. Comput. Math. 5 (2002), $95-126$.

[7] B. Huppert, Endliche Gruppen I, Springer-Verlag, New York-Heidelberg-Berlin, 1967.

[8] O. H. King, The subgroup structure of finite classical groups in terms of geometric configurations, in Surveys in combinatorics 2005, in London Math. Soc. Lecture Note Ser. 327, pp. 29-56 Cambridge Univ. Press, Cambridge, 2005.

[9] O. H. King, On subgroups of the special linear group containing the special unitary group, Geom. Dedicata 19 (1985), 297-310.

[10] O. H. King, On subgroups of the special linear group containing the special orthogonal group, J.Algebra 96 (1985), 178-193.

[11] P. Kleidman and M. Liebeck, The Subgroup Structure of the Finite Classical Groups, London Math. Soc. Lecture Notes Series 129, Cambridge University Press, Cambridge, 1990.

[12] L. G. Kovacs, Primitive subgroups of wreath products in product action, Proc. London Math. Soc. (3) 58 (1989), 306-322.

[13] F. Lübeck, Small degree representations of finite Chevalley groups in defining characteristic. LMS J. Comput. Math. 4 (2001), 135-169.

[14] C. E. Praeger and J. Saxl, Closures of finite primitive permutation groups, Bull. London Math. Soc. 24 (1992), 251-258.

[15] C. E. Praeger and J. Xu, A note on arc-transitive circulant digraphs, J. Group Theory, 12 (2009) 107-117. 
[16] E. Snapper and R. J. Troyer, Metric Affine Geometry, Academic Press, New York and London, 1971.

[17] M. Suzuki, Group Theory I, Springer-Verlag, New York-Heidelberg-Berlin, 1982.

[18] D. E. Taylor, The Geometry of the Classical Groups, Sigma Series in Pure Mathematics, Vol. 9, Heldermann-Verlag, Berlin, 1992.

[19] H. Wielandt, Permutation Groups Through Invariant Relations and Invariant Functions, Lecture Notes, Ohio State University, Columbus, 1969. Also publised in Wielandt, Helmut, Mathematische Werke/Mathematical works. Vol. 1. Group theory. Walter de Gruyter \& Co., Berlin, 1994, pp.237-296.

[20] Jing Xu, On closures of finite permutation groups, PhD Thesis, University of Western Australia, 2006.

[21] K. Zsigmondy, Zur Theorie der Potenzreste, Monatsh. für Math. und Phys. 3 (1892), 265-284. 